\title{
LARGE DEVIATIONS APPROXIMATIONS TO DISTRIBUTIONS OF THE TOTAL DISTANCE OF COMPOUND RANDOM WALKS WITH VON MISES DIRECTIONS
}

\author{
Riccardo Gatto \\ Submitted: April 2016 \\ Revised: July 2016
}

\begin{abstract}
This article considers the planar random walk where the direction taken by each consecutive step follows the von Mises distribution and where the number of steps of the random walk is determined by the class of inhomogeneous birth processs. Saddlepoint approximations to the distribution of the total distance covered by the random walk, i.e. of the length of the resultant vector of the individual steps, are proposed. Specific formulae are derived for the inhomogeneous Poisson process and for processes with linear contagion, which are the binomial and the negative binomial processes. A numerical example confirms the high accuracy of the proposed saddlepoint approximations.
\end{abstract}

\section{Key words and phrases}

Binomial, negative binomial and Poisson processes; circular distribution; defective density; resultant length; saddlepoint approximation.

The author thanks two anonymous Referees and an Associate Editor for various remarks which improved the quality of this article.

\section{Mathematics Subject Classification}

41A60 Asymptotic approximations, asymptotic expansions (steepest descent, etc.)

62H11 Directional data; spatial statistics

\section{Address}

Institute of Mathematical Statistics and Actuarial Science

Department of Mathematics and Statistics

University of Bern

Alpeneggstrasse 22, 3012 Bern, Switzerland

gatto@stat.unibe.ch, +41316318807

orcid.org/0000-0001-8374-6964 


\section{Introduction}

This article considers the random walk over the plane where the directions taken by the individual steps follow the isotropic and the von Mises circular distributions and where the total number of steps is determined by the class of inhomogeneous birth processes. A twodimensional direction can be represented by an angle, in radians for example, and a circular distribution is the probability distribution of a random angle, also called circular random variable; refer e.g. to Chapter 3 of Mardia and Jupp (2000). This type of random walk, also called random flight, appears in physics, see e.g. Barber and Ninham (1970), statistical mechanics, see e.g. Flory (1969), crystallography, see e.g. Srinivisan and Parthasarathy (1976), and in other disciplines such as atmospheric science, mathematical ecology, etc. The aim of the article is to provide a large deviations approximation to the distribution of the total distance covered by this compound random walk.

For this purpose we consider the saddlepoint approximation of asymptotic analysis, which is a large deviations method. It is substantially more accurate than limit normal or Edgeworth approximations, especially when computing very small tail probabilities. The outstanding accuracy of the saddlepoint approximation can be explained by the fact that it has bounded relative error, over the entire domain of the distribution. In fact saddlepoint approximations are useful for computing probability of rare events and compete well with techniques of rare event simulation, importance sampling essentially, because they do not require (computer intensive) Monte Carlo sampling. Two general references on saddlepoint approximations in statistics and probability are Field and Ronchetti (1990) and Jensen (1995a).

The random walks considered in this article are directed by the von Mises circular density, which is given by

$$
f(\theta \mid \mu, \kappa)=\frac{1}{2 \pi I_{0}(\kappa)} \mathrm{e}^{\kappa \cos (\theta-\mu)},
$$

$\forall \theta, \mu \in[0,2 \pi), \kappa \geq 0$. All angles in this article are expressed in the radian measure and are arbitrarily restricted to $[0,2 \pi)$. As usual, $I_{k}(z)=(2 \pi)^{-1} \int_{0}^{2 \pi} \cos k \theta \exp \{z \cos \theta\} d \theta, \forall z \in \mathbb{C}$, is the modified Bessel function $I$ of integer order $k$, see e.g. p. 376 of Abramowitz and Stegun (1972). The parameter $\mu$ is the location parameter and $\kappa$ is the concentration parameter. We denote this distribution as $\operatorname{vM}(\mu, \kappa)$ distribution. Note that $\kappa=0$ yields the circular uniform i.e. the isotropic distribution. We consider the von Mises distribution for the individual steps of the random walk, because it possesses several interesting properties, which can be found e.g. at Section 2.2.4 of Jammalamadaka and SenGupta (2001). The von Mises distribution is often considered as important as the normal distribution is for linear data and it is often called circular normal distribution. Both distributions possess many important properties. For example, the von Mises distribution maximizes Shannon's entropy among all circular densities with given first trigonometric moment, i.e. with fixed mean direction and mean resultant length. We recall that Shannon's entropy $-\int_{0}^{2 \pi} \log f(\theta) f(\theta) \mathrm{d} \theta$ is an appropriate measure of the uncertainty carried by the circular distribution with density $f$, whereas the first trigonometric moment (or Fourier coefficient) 
of this circular distribution is given by $\int_{0}^{2 \pi} \mathrm{e}^{\mathrm{i} \theta} f(\theta) \mathrm{d} \theta$. The maximum entropy principle states that, subject to known constraints, the distribution which best represents the current state of knowledge is the one with maximal entropy.

The results of this article extend previous results for random walks with fixed number of steps. Barakat (1973) provides a computational scheme for a Fourier series approximation to the density of the total distance of the isotropic random walk, with uniformly distributed step size. Exploiting results on Bessel functions, Kolesnik and Orsingher (2005) obtain the distribution of the random walk with isotropic directions and exponentially distributed step lengths. Let $\left\{N_{t}\right\}_{t \geq 0}$ be the homogeneous Poisson process of the number of direction changes of a particle moving with constant velocity. By interpreting the step length as the random elapsed time between two consecutive changes of direction of the particle, the position of the particle after $n$ steps becomes the position at time $t$, conditional on $\left\{N_{t}=n\right\}$. The unconditional distribution of the particle at time $t$ is then deduced from this conditional distribution. The multivariate version of this problem is analyzed in Orsingher and De Gregorio (2007); see also Stadje (1987, 1989) and Masoliver et al. (1993). Saddlepoint approximations for the isotropic and von Mises random walks with fixed number of steps are given in Jensen (1995), p. 162-165. Further, Weiss and Kiefer (1983) suggest a saddlepoint approximation for the projection of the isotropic random walk with fixed but unequal step sizes. Gatto and Mayer (2005) give a saddlepoint approximation for this projected random walk, but with exponential step size. Gatto and Jammalamadaka (2003) propose a saddlepoint approximation for the random walk with wrapped symmetric $\alpha$-stable directions.

The present article provides saddlepoint approximations for the planar isotropic and von Mises random flight with general inhomogeneous birth processes (which includes the homogeneous Poisson process mentioned in the previous paragraph) as well as for the isotropic random flight with fixed number of steps and exponential lengths. Precisely, Section 2 provides the saddlepoint approximation to the distribution of the total distance covered by random walk when of the number of steps is fixed: Section 2.1 considers the isotropic case, Section 2.2 the isotropic case with exponential step size and Section 2.3 considers the von Mises case. Section 3 provides the saddlepoint approximation for the total distance of the random flight when the number of steps is determined by an independent general counting process: the isotropic case is given in Section 3.1 and the von Mises case in Section 3.2. Section 4 considers three types of inhomogeneous birth processes: specific formulae for the Poisson process are derived in Section 4.1 and for the binomial and negative binomial processes in Section 4.2. Section 5 provides a numerical illustration of the high accuracy of the saddlepoint approximation, for the case of the compound Poisson random walk. Some final remarks are given in Section 6 . The notation $\mathbb{N}=\{0,1, \ldots\}$ is used. 


\section{Random walk distance under isotropy and von Mises directions}

This section considers the random walk with fixed number of steps: the case of uniform directions is considered in Section 2.1 and the case of von Mises directions is considered in Section 2.3. Saddlepoint approximations for the total length of the random walk are derived.

The saddlepoint approximation to the density of the mean of $n$ i.i.d. random variables was suggested by Daniels (1954), who derived an asymptotic expansion in powers of $n^{-1}$, as $n \rightarrow \infty$. As mentioned in the introduction, the leading term of the saddlepoint expansion possesses a relative error of the order $n^{-1}$, at any point of the support of the density, that is over the large deviations region of the mean. In comparison, the normal approximation possesses only an absolute error of the larger order $n^{-1 / 2}$ and its validity is restricted to points which converge towards the center of the distribution at rate $n^{-1 / 2}$, namely over normal deviations regions. Consequently, the saddlepoint approximation is very adequate for approximating very small tail probabilities, e.g. of the order of $10^{-6}$, and even with very small samples sizes, e.g. $n=4$. In addition to the general references mentioned in the introduction, short reviews can be found in Jensen (1995b), Field and Tingley (1997) and Gatto (2015), for example.

The saddlepoint approximations obtained in this section are generalized to random walks with random number steps, i.e. compound random walks, in the next sections.

\section{$2.1 \quad$ Isotropic case}

Let $n \in \mathbb{N} \backslash\{0\}$ and $\theta_{1}, \ldots, \theta_{n}$ be independent and uniformly distributed viz. isotropic circular random variables over the probability space $(\Omega, \mathcal{F}, \mathrm{P})$ and taking values in $[0,2 \pi)$. Denote

$$
C_{n}=\sum_{j=1}^{n} \cos \theta_{j}, S_{n}=\sum_{j=1}^{n} \sin \theta_{j} \text { and } R_{n}\left(\cos \mu_{n}, \sin \mu_{n}\right)=\left(C_{n}, S_{n}\right)
$$

the polar representation, where $R_{n}$ takes values in $[0, n]$ and $\mu_{n}$ in $[0,2 \pi)$. Let $h_{n}$ be the density of $\left(C_{n}, S_{n}\right)$ and $g_{n}$ be the one of $\left(R_{n}, \mu_{n}\right)$. Thus

$$
g_{n}(r, \theta)=r h_{n}(r \cos \theta, r \sin \theta), \quad \forall r \in(0, n], \theta \in[0,2 \pi) .
$$

We are interested in the distribution of the resultant length $R_{n}$ under isotropy. The next lemma plays a central role in this article.

Lemma 2.1 (First factorization lemma). Let $q_{n}$ be the density of $R_{n}$ and $g_{n}$ be the joint density $\left(R_{n}, \mu_{n}\right)$, under isotropy. Then

$$
g_{n}(r, \theta)=\frac{1}{2 \pi} q_{n}(r), \forall r \in(0, n], \theta \in[0,2 \pi) .
$$


In fact the converse holds as well, as the circular uniform or isotropic distribution is characterized by the independence of $R_{n}$ and $\mu_{n}$ and isotropy of $\mu_{n}$, within absolutely continuous distributions and for $n \geq 2$, see Kent et al. (1979). We can note (to emphasize the analogy between von Mises and normal distributions) that the analogous factorization holds with the normal distribution, in terms of sample mean and sample variance. We denote $A=I_{1} / I_{0}$, which is a continuous increasing function from $[0, \infty)$ onto $[0,1)$ and thus a distribution function, and we denote by $A^{(-1)}$ its inverse function i.e. the quantile function. We also denote by $\mathrm{E}$ the expectation functional of random variables over $(\Omega, \mathcal{F}, \mathrm{P})$. We can now show the following proposition.

Proposition 2.2. The saddlepoint approximation to $q_{n}(r)$, the density of $R_{n}$ under isotropy, is given by

$$
\tilde{q}_{n}(r)=\frac{1}{n \sigma(\bar{u})} I_{0}^{n}(\bar{u}) r \mathrm{e}^{-\bar{u} r}
$$

where

$$
\bar{u}=A^{(-1)}\left(\frac{r}{n}\right)
$$

which can be evaluated explicitly with

$$
A^{(-1)}(x)=\left\{\begin{array}{lr}
2 x+x^{3}+0.83 x^{5}, & \text { if } 0 \leq x<0.53 \\
-0.4+1.39 x+0.43(1-x)^{-1}, & \text { if } 0.53 \leq x<0.85 \\
\left(x^{3}-4 x^{2}+3 x\right)^{-1}, & \text { if } 0.85 \leq x<1
\end{array}\right.
$$

$\forall r \in(0, n]$, and where $\sigma(\bar{u})$ is given by

$$
\sigma^{2}(u)= \begin{cases}\frac{1}{4}, & \text { if } u=0 \\ \frac{A(u) A^{\prime}(u)}{u}, & \text { if } u>0 .\end{cases}
$$

$\forall r \in(0, n], q_{n}(r)=\tilde{q}_{n}(r)\left\{1+\mathrm{O}\left(n^{-1}\right)\right\}$, as $n \rightarrow \infty$.

Let $c_{n}^{-1}=\int_{0}^{n} \tilde{q}_{n}(r) \mathrm{d} r$, then $c_{n} \tilde{q}_{n}(r)$ is the normalized saddlepoint approximation to $q_{n}(r)$ and $q_{n}(r)=c_{n} \tilde{q}_{n}(r)\left\{1+\mathrm{O}\left(n^{-3 / 2}\right)\right\}$, for $r$ over normal deviation regions, as $n \rightarrow \infty$.

Also, $\bar{u}$ is a continuous increasing function of $r$ over $(0, n]$ with $\bar{u} \rightarrow 0$, as $r \rightarrow 0$.

Proof The saddlepoint approximation to $g_{n}$, the joint density $\left(R_{n}, \mu_{n}\right)$, can be obtained as follows. The moment generating function (m.g.f.) of $\left(C_{1}, S_{1}\right)$ is given by

$$
M\left(v_{1}, v_{2}\right)=\mathrm{E}\left[\exp \left\{v_{1} C_{1}+v_{2} S_{1}\right\}\right]=\frac{1}{2 \pi} \int_{0}^{2 \pi} \mathrm{e}^{u \cos (\theta-\nu)} \mathrm{d} \theta=I_{0}(u)
$$

where $\left(v_{1}, v_{2}\right)=u(\cos \nu, \sin \nu) \in \mathbb{R}^{2}$. The cumulant generating function (c.g.f.) is $K=$ $\log M$. Thus, the c.g.f. of $\left(C_{n}, S_{n}\right)$ is $n K$. The saddlepoint at $(c, s)=r(\cos \theta, \sin \theta) \in[0, n]^{2}$ is the solution in $\left(v_{1}, v_{2}\right)=u(\cos \nu, \sin \nu)$ of

$$
\left(\begin{array}{c}
\frac{\partial}{\partial v_{1}} n K\left(v_{1}, v_{2}\right) \\
\frac{\partial}{\partial v_{2}} n K\left(v_{1}, v_{2}\right)
\end{array}\right)=\left(\begin{array}{c}
c \\
s
\end{array}\right) \Longleftrightarrow A(u)\left(\begin{array}{c}
\cos \nu \\
\sin \nu
\end{array}\right)=\frac{r}{n}\left(\begin{array}{c}
\cos \theta \\
\sin \theta
\end{array}\right) \Longleftrightarrow A(u)=\frac{r}{n}, \nu=\theta .
$$


Thus the saddlepoint in polar coordinates is given by $(\bar{u}, \bar{\nu})=\left(A^{(-1)}(r / n), \theta\right)$. Note that at $(c, s)=\mathrm{E}\left[\left(C_{n}, S_{n}\right)\right]=(0,0)$, both $\theta$ and $\bar{\nu}$ are undetermined, whereas $\bar{u}=0$.

The determinant of the Hessian matrix of $K$ at $(u \cos \nu, u \sin \nu)$ can be obtained, by computer algebra, as in (4), refer also to p. 163 of Jensen (1995a) and to p. 354 of Gatto and Jammalamadaka (2003) for the case $u=0$. It can be seen (from e.g. the second and fourth recurrence relations of 9.6.26 at p. 376 of Abramowitz and Stegun, 1972) that the derivative $A^{\prime}$ can be obtained by Riccati's differential equation

$$
A^{\prime}(u)=1-A^{2}(u)-\frac{A(u)}{u}, \forall u>0 .
$$

Thus the saddlepoint approximation to $h_{n}(c, s)$, the density of $\left(C_{n}, S_{n}\right)$, is given by

$$
\begin{aligned}
\tilde{h}_{n}(c, s) & =\{2 \pi n \sigma(\bar{u})\}^{-1} \exp \{n K(\bar{u} \cos \bar{\nu}, \bar{u} \sin \bar{\nu})-\bar{u} \cos \bar{\nu} r \cos \theta-\bar{u} \sin \bar{\nu} r \sin \theta\} \\
& =\{2 \pi n \sigma(\bar{u})\}^{-1} I_{0}^{n}(\bar{u}) \mathrm{e}^{-\bar{u} r}
\end{aligned}
$$

where $h_{n}(c, s)=\tilde{h}_{n}(c, s)\left\{1+\mathrm{O}\left(n^{-1}\right)\right\}, \forall(c, s) \in[0, n]^{2}$, as $n \rightarrow \infty$; see e.g. Section 6.5 of Barndorff-Nielsen and Cox (1989). Thus at the expectation, $\tilde{h}_{n}(0,0)=(n \pi)^{-1}$. Transforming to polar coordinates leads to the saddlepoint approximation

$$
\tilde{g}_{n}(r, \theta)=\frac{1}{2 \pi n \sigma(\bar{u})} I_{0}^{n}(\bar{u}) r \mathrm{e}^{-\bar{u} r}
$$

where $g_{n}(r, \theta)=\tilde{g}_{n}(r, \theta)\left\{1+\mathrm{O}\left(n^{-1}\right)\right\}$, holds $\forall r \in(0, n], \theta \in[0,2 \pi)$, as $n \rightarrow \infty$. This last approximation and the First factorization lemma yield the claimed saddlepoint approximation. The approximation to $A^{(-1)}$ given in (3) is proposed by Best and Fisher (1981) for maximum likelihood estimation (and used by Gatto and Mayer, 2005, for solving the saddlepoint equation). Concerning the relative error $\mathrm{O}\left(n^{-3 / 2}\right)$ over normal deviation regions, one can refer for example to p. 31 of Field and Ronchetti (1990).

Note that in 1906 Kluyver expressed the exact density of the resultant length under isotropy as

$$
q_{n}(r)=r \int_{0}^{\infty} J_{0}(r t) J_{0}^{n}(t) t \mathrm{~d} t, \quad \forall r \in(0, n]
$$

where $J_{\nu}(z)=(z / 2)^{\nu} /\{\sqrt{\pi} \Gamma(\nu+1 / 2)\} \int_{0}^{\pi} \mathrm{e}^{\mathrm{i} z \cos \theta} \sin ^{2 \nu} \theta \mathrm{d} \theta, \forall z \in \mathbb{C}, \Re \nu>-1 / 2$, is the Bessel function of the first kind, see e.g. p. 360 of Abramowitz and Stegun (1972). Thus, Proposition 2.2 provides the saddlepoint approximation to Kluyver's integral (7). Note that the infinite integration domain and the oscillating integrand of (7) render numerical integration difficult.

\section{$2.2 \quad$ Isotropic case with exponential step size}

Although this article is mainly concerned with random walks with fixed step size, this section briefly presents the extension of the isotropic random walk of Section 2.1 to the 
situation where the step sizes are exponentially distributed random variables. The beauty of the exponential length is that it yields a simpler saddlepoint approximation with a closed-form expression for the saddlepoint. As mentioned in the introduction, Gatto and Mayer (2005) consider a one-dimensional projection of this random walk. The random walk has $n \in \mathbb{N} \backslash\{0\}$ steps with independent and isotropic directions $\theta_{1}, \ldots, \theta_{n}$ and independent lengths $X_{1}, \ldots, X_{n}$ with the exponential distribution $l(x)=\gamma \mathrm{e}^{-\gamma x}, \forall \gamma, x>0$. Angles and lengths are independent. All random variables are defined over the probability space $(\Omega, \mathcal{F}, P)$ and $E$ denotes the associated expectation functional. We denote

$$
\hat{C}_{n}=\sum_{j=1}^{n} X_{j} \cos \theta_{j}, \hat{S}_{n}=\sum_{j=1}^{n} X_{j} \sin \theta_{j} \text { and } \hat{R}_{n}\left(\cos \hat{\mu}_{n}, \sin \hat{\mu}_{n}\right)=\left(\hat{C}_{n}, \hat{S}_{n}\right)
$$

the polar representation, where $\hat{R}_{n}$ takes values in $[0, \infty)$ and $\hat{\mu}_{n}$ in $[0,2 \pi)$. Let $\hat{h}_{n}$ be the density of $\left(\hat{C}_{n}, \hat{S}_{n}\right)$ and $\hat{g}_{n}$ be the one of $\left(\hat{R}_{n}, \hat{\mu}_{n}\right)$. Thus

$$
\hat{g}_{n}(r, \theta)=r \hat{h}_{n}(r \cos \theta, r \sin \theta), \quad \forall r \in(0, \infty), \theta \in[0,2 \pi) .
$$

The following generalization the First factorization lemma is required.

Lemma 2.3 (Generalized first factorization lemma). Let $\hat{q}_{n}$ be the density of $\hat{R}_{n}$ and $\hat{g}_{n}$ be the joint density $\left(\hat{R}_{n}, \hat{\mu}_{n}\right)$, under isotropy. Then

$$
\hat{g}_{n}(r, \theta)=\frac{1}{2 \pi} \hat{q}_{n}(r), \forall r>0, \theta \in[0,2 \pi) .
$$

Proof The notation $V_{1} \sim V_{2}$ means that $V_{1}$ and $V_{2}$ have same distribution and $V_{1} \mid W_{1} \sim$ $V_{2} \mid W_{2}$ means that the conditional distribution of $V_{1}$ given $W_{1}$ is equal to the one of $V_{2}$ given $W_{2}$.

Let $\alpha \in[0,2 \pi)$ and denote

$$
\begin{array}{r}
\hat{C}_{n}(\alpha)=\sum_{j=1}^{n} X_{j} \cos \left(\theta_{j}+\alpha\right), \hat{S}_{n}(\alpha)=\sum_{j=1}^{n} X_{j} \sin \left(\theta_{j}+\alpha\right) \\
\text { and } \hat{R}_{n}(\alpha)\left(\cos \hat{\mu}_{n}(\alpha), \sin \hat{\mu}_{n}(\alpha)\right)=\left(\hat{C}_{n}(\alpha), \hat{S}_{n}(\alpha)\right)
\end{array}
$$

the polar representation, where $\hat{R}_{n}(\alpha)$ takes values in $[0, \infty)$ and $\hat{\mu}_{n}(\alpha)$ in $[0,2 \pi)$. The invariances $\hat{R}_{n}(\alpha)=\hat{R}_{n}$ and $\hat{\mu}_{n}(\alpha)=\hat{\mu}_{n}+\alpha$ can be directly understood. Isotropy implies

$$
\left(\left(X_{1}, \theta_{1}\right), \ldots,\left(X_{n}, \theta_{n}\right)\right) \sim\left(\left(X_{1}, \theta_{1}+\alpha\right), \ldots,\left(X_{n}, \theta_{n}+\alpha\right)\right),
$$

which together with the just stated invariances implies

$$
\left(\hat{\mu}_{n}, \hat{R}_{n}\right) \sim\left(\hat{\mu}_{n}+\alpha, \hat{R}_{n}\right) .
$$

Consequently,

$$
\hat{\mu}_{n} \sim \hat{\mu}_{n}+\alpha \text { and } \hat{\mu}_{n}\left|\hat{R}_{n} \sim \hat{\mu}_{n}+\alpha\right| \hat{R}_{n}
$$

meaning that $\hat{\mu}_{n}$ and $\hat{\mu}_{n} \mid \hat{R}_{n}$ are isotropic. This in turn implies $\hat{\mu}_{n} \sim \hat{\mu}_{n} \mid \hat{R}_{n}$, viz. $\hat{\mu}_{n}$ and $\hat{R}_{n}$ are independent.

We can now show the following result. 
Proposition 2.4. The saddlepoint approximation to $\hat{q}_{n}(r)$, the density of $R_{n}$ under isotropy and exponential step lengths, is given by

$$
\tilde{\hat{q}}_{n}(r)=\frac{1}{n \hat{\sigma}(\bar{u})}\left(\frac{\gamma}{\sqrt{\gamma^{2}-\bar{u}^{2}}}\right)^{n} r \mathrm{e}^{-\bar{u} r},
$$

where

$$
\bar{u}=\frac{-n+\sqrt{n^{2}+4 r^{2} \gamma^{2}}}{2 r}
$$

$\forall r>0$, and where $\hat{\sigma}(\bar{u})$ is given by

$$
\hat{\sigma}^{2}(u)=\frac{\gamma^{2}+u^{2}}{\left(\gamma^{2}-u^{2}\right)^{3}} .
$$

$\forall r>0, \hat{q}_{n}(r)=\tilde{\hat{q}}_{n}(r)\left\{1+\mathrm{O}\left(n^{-1}\right)\right\}$, as $n \rightarrow \infty$.

Let $\hat{c}_{n}^{-1}=\int_{0}^{n} \tilde{\hat{q}}_{n}(r) \mathrm{d} r$, then $\hat{c}_{n} \tilde{\hat{q}}_{n}(r)$ is the normalized saddlepoint approximation to $\hat{q}_{n}(r)$ and $\hat{q}_{n}(r)=\hat{c}_{n} \hat{\hat{q}}_{n}(r)\left\{1+\mathrm{O}\left(n^{-3 / 2}\right)\right\}$, for $r$ over normal deviation regions, as $n \rightarrow \infty$.

Also, $\bar{u}$ is a continuous increasing function of $r$ over $(0, \infty)$ with $\bar{u} \rightarrow 0$, as $r \rightarrow 0$.

Proof By using (5), we can compute the m.g.f. of $X_{1}\left(\cos \theta_{1}, \sin \theta_{1}\right)$ as

$$
\begin{aligned}
\hat{M}\left(v_{1}, v_{2}\right) & =\mathrm{E}\left[\mathrm{E}\left[\exp \left\{v_{1} \hat{C}_{1}+v_{2} \hat{S}_{1}\right\} \mid X_{1}\right]\right] \\
& =\mathrm{E}\left[\hat{M}\left(v_{1} X_{1}, v_{2} X_{1}\right)\right] \\
& =\int_{0}^{\infty} I_{0}(u x) l(x) \mathrm{d} x,
\end{aligned}
$$

where $\left(v_{1}, v_{2}\right)=u(\cos \nu, \sin \nu) \in \mathbb{R}^{2}$. By replacing $I_{0}$ the ascending series

$$
I_{0}(z)=\sum_{j=0}^{\infty}\left(\frac{\left(\frac{z}{2}\right)^{j}}{j !}\right)^{2}, \forall z \in \mathbb{C},
$$

see e.g. p. 375 of Abramowitz and Stegun (1972), we obtain

$$
\hat{M}\left(v_{1}, v_{2}\right)=\sum_{j=0}^{\infty}\left(\frac{u^{j}}{2^{j} j !}\right)^{2} \mathrm{E}\left[X_{1}^{2 j}\right] .
$$

As exponentiality is assumed, $\mathrm{E}\left[X_{1}^{j}\right]=j ! / \gamma^{j}, \forall j \in \mathbb{N}$, and we obtain

$$
\hat{M}\left(v_{1}, v_{2}\right)=\sum_{j=0}^{\infty}\left(\frac{u^{j}}{(2 \gamma)^{j} j !}\right)^{2}(2 j) ! .
$$

The primitive of this series is the series of $\gamma \arcsin (u / \gamma)$, which is also equal to $\int \gamma / \sqrt{\gamma^{2}-u^{2}} \mathrm{~d} u$. This implies that, $\forall\left(v_{1}, v_{2}\right) \in \mathbb{R}^{2}$ such that $u^{2}<\gamma^{2}$,

$$
\hat{M}\left(v_{1}, v_{2}\right)=\frac{\gamma}{\sqrt{\gamma^{2}-u^{2}}}
$$


and the c.g.f. of $\left(\hat{C}_{n}, \hat{S}_{n}\right)$ is

$$
n \hat{K}\left(v_{1}, v_{2}\right)=n \log \hat{M}\left(v_{1}, v_{2}\right)=n \log \frac{\gamma}{\sqrt{\gamma^{2}-u^{2}}} .
$$

The saddlepoint at $(c, s)=r(\cos \theta, \sin \theta) \in[0, \infty)^{2}$ is the solution in $\left(v_{1}, v_{2}\right)=u(\cos \nu, \sin \nu)$ of

$$
\left(\begin{array}{c}
\frac{\partial}{\partial v_{1}} n \hat{K}\left(v_{1}, v_{2}\right) \\
\frac{\partial}{\partial v_{2}} n \hat{K}\left(v_{1}, v_{2}\right)
\end{array}\right)=\left(\begin{array}{c}
c \\
s
\end{array}\right) \Longleftrightarrow \frac{u}{\gamma^{2}-u^{2}}\left(\begin{array}{c}
\cos \nu \\
\sin \nu
\end{array}\right)=\frac{r}{n}\left(\begin{array}{c}
\cos \theta \\
\sin \theta
\end{array}\right) \Longleftrightarrow \frac{u}{\gamma^{2}-u^{2}}=\frac{r}{n}, \nu=\theta \text {. }
$$

Thus the saddlepoint in polar coordinates is given by $\bar{u}$ as in (8) and $\bar{\nu}=\theta$. The determinant of the Hessian matrix of $\hat{K}$ at $(u \cos \nu, u \sin \nu)$ is given in (9). Thus the saddlepoint approximation to $\hat{h}_{n}(c, s)$, the density of $\left(\hat{C}_{n}, \hat{S}_{n}\right)$, is given by

$$
\begin{aligned}
\tilde{\hat{h}}_{n}(c, s) & =\{2 \pi n \hat{\sigma}(\bar{u})\}^{-1} \exp \{n \hat{K}(\bar{u} \cos \bar{\nu}, \bar{u} \sin \bar{\nu})-\bar{u} \cos \bar{\nu} r \cos \theta-\bar{u} \sin \bar{\nu} r \sin \theta\} \\
& =\{2 \pi n \hat{\sigma}(\bar{u})\}^{-1}\left(\frac{\gamma}{\sqrt{\gamma^{2}-\bar{u}^{2}}}\right)^{n} \mathrm{e}^{-\bar{u} r}
\end{aligned}
$$

where $\hat{h}_{n}(c, s)=\tilde{\hat{h}}_{n}(c, s)\left\{1+\mathrm{O}\left(n^{-1}\right)\right\}, \forall(c, s) \in[0, \infty)^{2}$, as $n \rightarrow \infty$. The saddlepoint approximation is polar coordinates is

$$
\tilde{\hat{g}}_{n}(r, \theta)=\frac{1}{2 \pi n \hat{\sigma}(\bar{u})}\left(\frac{\gamma}{\sqrt{\gamma^{2}-\bar{u}^{2}}}\right)^{n} r \mathrm{e}^{-\bar{u} r}
$$

where $\hat{g}_{n}(r, \theta)=\tilde{\hat{g}}_{n}(r, \theta)\left\{1+\mathrm{O}\left(n^{-1}\right)\right\}$, holds $\forall r \in(0, \infty), \theta \in[0,2 \pi)$, as $n \rightarrow \infty$. This and the Generalized first factorization lemma conclude the proof.

\subsection{Von Mises case}

Assume now that the $\operatorname{vM}(\mu, \kappa)$ distribution with density (1) for $\theta_{1}, \ldots, \theta_{n}$. Let us denote by $q_{\kappa, n}$ the density of the resultant length $R_{n}$, under the $\operatorname{vM}(\mu, \kappa)$ distribution. Thus $q_{0, n}=q_{n}$. The next lemma is due to Greenwood and Durand (1955); see also p. 72 of Jammalamadaka and SenGupta (2001).

Lemma 2.5 (Tilting lemma). The densities of $R_{n}$ under isotropy and under the $\operatorname{vM}(\mu, \kappa)$ distribution, viz. $q_{n}$ and $q_{\kappa, n}$ respectively, satisfy the relation

$$
q_{\kappa, n}(r)=\frac{I_{0}(\kappa r)}{I_{0}^{n}(\kappa)} q_{n}(r),
$$

$\forall r \in(0, n]$.

Let us define

$$
B(x, y)=\log \frac{I_{0}(x)}{I_{0}(y)}, \quad \forall x, y \geq 0 .
$$

Proposition 2.2 and Lemma 2.5 yield the following result. 
Proposition 2.6. The saddlepoint approximation to $q_{\kappa, n}(r)$, the density of $R_{n}$ under the $\operatorname{vM}(\mu, \kappa)$ distribution, is given by

$$
\tilde{q}_{\kappa, n}(r)=\frac{I_{0}(\kappa r)}{n \sigma(\bar{u})} \mathrm{e}^{n B(\bar{u}, \kappa)} r \mathrm{e}^{-\bar{u} r}
$$

where $\bar{u}$ is the saddlepoint given by (2), which is evaluated by (3), and where $\sigma$ is given by (4), $\forall r \in(0, n] . \forall r \in(0, n], q_{\kappa, n}(r)=\tilde{q}_{\kappa, n}(r)\left\{1+\mathrm{O}\left(n^{-1}\right)\right\}$, as $n \rightarrow \infty$.

Let $c_{\kappa, n}^{-1}=\int_{0}^{n} \tilde{q}_{\kappa, n}(r) \mathrm{d} r$, then $c_{\kappa, n} \tilde{q}_{\kappa, n}(r)$ is the normalized saddlepoint approximation to $q_{\kappa, n}(r)$ and $q_{\kappa, n}(r)=c_{\kappa, n} \tilde{q}_{\kappa, n}(r)\left\{1+\mathrm{O}\left(n^{-3 / 2}\right)\right\}$, for $r$ over normal deviation regions, as $n \rightarrow \infty$.

$\forall r \in(0, n]$.

Note that $q_{\kappa, n}$ and $\tilde{q}_{\kappa, n}$ do not depend on $\mu$. Propositions 2.2 and 2.6, which can also found at p. 163 of Jensen (1995a), essentially, are generalized to the compound random walk in the next sections.

\section{Compound random walk distance under isotropy and von Mises directions}

This section considers the compound random walk, i.e. the random walk with a random number of steps. Section 3.1 deals with isotropic individual steps and the generalization to von Mises steps is given in Section 3.2. Saddlepoint approximations for the total length of the compound random walk are derived. Let $\left\{N_{t}\right\}_{t \geq 0}$ be a counting process, i.e. an a.s. nondecreasing $\mathbb{N}$-valued process, defined over $(\Omega, \mathcal{F}, \mathrm{P})$. Assume $N_{0}=0$ a.s.

\subsection{Isotropic case}

Define over $(\Omega, \mathcal{F}, \mathrm{P})$ the $[0,2 \pi)$-valued circular random variables $\theta_{1}, \theta_{2}, \ldots$ as independent, isotropic and independent of $\left\{N_{t}\right\}_{t \geq 0}$. Let $t \geq 0$ be any fixed time horizon and define $p_{n}(t)=\mathrm{P}\left[N_{t}=n\right], \forall n \in \mathbb{N}$,

$$
C_{t}^{*}=\sum_{j=1}^{N_{t}} \cos \theta_{j}, S_{t}^{*}=\sum_{j=1}^{N_{t}} \sin \theta_{j} \text { and } R_{t}^{*}\left(\cos \mu_{t}^{*}, \sin \mu_{t}^{*}\right)=\left(C_{t}^{*}, S_{t}^{*}\right),
$$

the polar representation, where $C_{t}^{*}=S_{t}^{*}=R_{t}^{*}=0$ over $\left\{N_{t}=0\right\}$ and $\mu_{t}^{*}$ is irrelevant over $\left\{N_{t}=0\right\}$. Thus $R_{t}^{*}$ takes values in $[0, \infty)$ and $\mu_{t}^{*}$ in $[0,2 \pi)$. The distribution of the compound resultant length $R_{t}^{*}$ under isotropy is desired. Let $h_{t}^{*}$ be the density of $\left(C_{t}^{*}, S_{t}^{*}\right)$ conditional on $\left\{N_{t}>0\right\}$, and $g_{t}^{*}$ be the density of $\left(R_{t}^{*}, \mu_{t}^{*}\right)$ conditional on $\left\{N_{t}>0\right\}$. Then we have

$$
g_{t}^{*}(r, \theta)=r h_{t}^{*}(r \cos \theta, r \sin \theta), \forall r>0, \theta \in[0,2 \pi)
$$

The following lemma implies that, under isotropy, $R_{t}^{*}$ and $\mu_{t}^{*}$ are independent. 
Lemma 3.1 (Second factorization lemma). Let $q_{t}^{*}$ be the density of $R_{t}^{*}$ conditional on $\left\{N_{t}>0\right\}$ and $g_{t}^{*}$ be the joint density $\left(R_{t}^{*}, \mu_{t}^{*}\right)$ conditional on $\left\{N_{t}>0\right\}$, under isotropy. Then

$$
g_{t}^{*}(r, \theta)=\frac{1}{2 \pi} q_{t}^{*}(r), \quad \forall r>0, \theta \in[0,2 \pi) .
$$

Proof Let $r>0, \theta \in[0,2 \pi)$, then from the First factorization lemma,

$$
\begin{aligned}
g_{t}^{*}(r, \theta) \mathrm{d} r \mathrm{~d} \theta & =\mathrm{P}\left[R_{t}^{*} \in(r, r+\mathrm{d} r), \mu_{t}^{*} \in(\theta, \theta+\mathrm{d} \theta) \mid N_{t}>0\right] \\
& =\frac{\sum_{n=0}^{\infty} \mathrm{P}\left[R_{t}^{*} \in(r, r+\mathrm{d} r), \mu_{t}^{*} \in(\theta, \theta+\mathrm{d} \theta), N_{t}=n, N_{t}>0\right]}{\mathrm{P}\left[N_{t}>0\right]} \\
& =\sum_{n=1}^{\infty} g_{n}(r, \theta) \mathrm{d} r \mathrm{~d} \theta \frac{p_{n}(t)}{1-p_{0}(t)} \\
& =\sum_{n=1}^{\infty} \frac{1}{2 \pi} q_{n}(r) \mathrm{d} r \mathrm{~d} \theta \frac{p_{n}(t)}{1-p_{0}(t)} \\
& =\frac{\mathrm{d} \theta}{2 \pi} \mathrm{P}\left[R_{t}^{*} \in(r, r+\mathrm{d} r) \mid N_{t}>0\right] \\
& =\frac{1}{2 \pi} q_{t}^{*}(r) \mathrm{d} r \mathrm{~d} \theta .
\end{aligned}
$$

For convenience, denote by $N_{t}^{0}$ the zero-truncation of $N_{t}$. Precisely, set $N_{t}^{0}=N_{t}$ over $\left\{N_{t}>0\right\}$ and renormalize its distribution. Thus $N_{t}^{0}$ has the conditional distribution of $N_{t}$ given $\left\{N_{t}>0\right\}$ and we define

$$
p_{n}^{0}(t)=\mathrm{P}\left[N_{t}^{0}=n\right]=\frac{p_{n}(t)}{1-p_{0}(t)}, \quad \forall n \in \mathbb{N} \backslash\{0\} .
$$

Therefore, by integrating with respect to $\theta$ the expression after the fourth equality in the proof of Lemma 3.1, we obtain

$$
q_{t}^{*}(r)=\sum_{n=1}^{\infty} q_{n}(r) \frac{p_{n}(t)}{1-p_{0}(t)}=\sum_{n=1}^{\infty} q_{n}(r) p_{n}^{0}(t), \forall r>0,
$$

for which the saddlepoint approximation is sought.

Proposition 3.2. The saddlepoint approximation to $q_{t}^{*}(r)$, the conditional density of $R_{t}^{*}$ given $\left\{N_{t}>0\right\}$, under isotropy, is given by

$$
\tilde{q}_{t}^{*}(r)=\sigma_{t}^{-1}(\bar{u}) M_{N_{t}^{0}}\left(\log I_{0}(\bar{u})\right) r \mathrm{e}^{-\bar{u} r}
$$

and the saddlepoint approximation to the defective density of $R_{t}^{*}$ is given by

$$
\tilde{q}_{t}^{*}(r)\left\{1-p_{0}(t)\right\},
$$

where $\bar{u}>0$ is the solution in $u$ of

$$
K_{N_{t}^{0}}^{\prime}\left(\log I_{0}(u)\right) A(u)-r=0
$$


$\forall r>0$, and where $\sigma_{t}(\bar{u})$ is given by the formula

$$
\sigma_{t}^{2}(u)=\frac{A(u)}{u} K_{N_{t}^{0}}^{\prime}\left(\log I_{0}(u)\right)\left\{K_{N_{t}^{0}}^{\prime}\left(\log I_{0}(u)\right) A^{\prime}(u)+K_{N_{t}^{0}}^{\prime \prime}\left(\log I_{0}(u)\right) A^{2}(u)\right\},
$$

$\forall u>0$, where $A^{\prime}$ can be evaluated with (6), and by the formula

$$
\lim _{u \rightarrow 0} \sigma_{t}^{2}(u)=\frac{1}{4} \mathrm{E}^{2}\left[N_{t} \mid N_{t}>0\right]
$$

Let $c_{t}^{*-1}=\int_{0}^{\infty} \tilde{q}_{t}^{*}(r) \mathrm{d} r$, then $c_{t}^{*} \tilde{q}_{t}^{*}(r)$ is the normalized saddlepoint approximation to $q_{t}^{*}(r), \forall r>0$.

Also, $\bar{u}$ is a continuous increasing function of $r$ over $(0, \infty)$ with $\bar{u} \rightarrow 0$, as $r \rightarrow 0$.

Proof The m.g.f. of $\left(C_{t}^{*}, S_{t}^{*}\right)$ conditional on $\left\{N_{t}>0\right\}$, is given by

$$
\begin{aligned}
M_{C_{t}^{*}, S_{t}^{*}}\left(v_{1}, v_{2}\right) & =\mathrm{E}\left[\mathrm{e}^{v_{1} C_{t}^{*}+v_{2} S_{t}^{*}} \mid N_{t}>0\right] \\
& =\left(\mathrm{P}\left[N_{t}>0\right]\right)^{-1} \mathrm{E}\left[\mathrm{E}\left[\mathrm{e}^{v_{1} C_{t}^{*}+v_{2} S_{t}^{*}} \mathrm{I}\left\{N_{t}>0\right\} \mid N_{t}\right]\right] \\
& =\left(\mathrm{P}\left[N_{t}>0\right]\right)^{-1} \mathrm{E}\left[\mathrm{E}\left[\mathrm{e}^{v_{1} C_{t}^{*}+v_{2} S_{t}^{*}} \mid N_{t}\right] \mathrm{I}\left\{N_{t}>0\right\}\right] \\
& =\mathrm{E}\left[M^{N_{t}}\left(v_{1}, v_{2}\right) \mid N_{t}>0\right] \\
& =\mathrm{E}\left[M^{N_{t}^{0}}\left(v_{1}, v_{2}\right)\right] \\
& =M_{N_{t}^{0}}\left(\log M\left(v_{1}, v_{2}\right)\right) \\
& =M_{N_{t}^{0}}\left(\log I_{0}(u)\right),
\end{aligned}
$$

where $\left(v_{1}, v_{2}\right)=u(\cos \nu, \sin \nu) \in \mathbb{R}^{2}$ and $M_{N_{t}^{0}}$ denotes the m.g.f. of $N_{t}^{0}$. Denoting $K_{N_{t}^{0}}=$ $\log M_{N_{t}^{0}}$ and $K_{C_{t}^{*}, S_{t}^{*}}=\log M_{C_{t}^{*}, S_{t}^{*}}$ the c.g.f., (13) gives

$$
K_{C_{t}^{*}, S_{t}^{*}}\left(v_{1}, v_{2}\right)=K_{N_{t}^{0}}\left(\log I_{0}(u)\right) .
$$

Thus, the saddlepoint at $(c, s)=r(\cos \theta, \sin \theta) \in \mathbb{R}^{2}$ is the solution in $\left(v_{1}, v_{2}\right)=u(\cos \nu, \sin \nu)$ of

$$
\begin{aligned}
\left(\begin{array}{c}
\frac{\partial}{\partial v_{1}} K_{C_{t}^{*}, S_{t}^{*}}\left(v_{1}, v_{2}\right) \\
\frac{\partial}{\partial v_{2}} K_{C_{t}^{*}, S_{t}^{*}}\left(v_{1}, v_{2}\right)
\end{array}\right)= & \left(\begin{array}{c}
c \\
s
\end{array}\right) \Longleftrightarrow K_{N_{t}^{0}}^{\prime}\left(\log I_{0}(u)\right) A(u)\left(\begin{array}{c}
\cos \nu \\
\sin \nu
\end{array}\right)=r\left(\begin{array}{c}
\cos \theta \\
\sin \theta
\end{array}\right) \\
& \Longleftrightarrow K_{N_{t}^{0}}^{\prime}\left(\log I_{0}(u)\right) A(u)=r, \nu=\theta
\end{aligned}
$$

Denoting the saddlepoint in polar coordinates as $(\bar{u}, \bar{\theta})$, we have that $\bar{u}$ is the implicit solution of the first equality in (14) and $\bar{\nu}=\theta$. Note that at $(c, s)=\mathrm{E}\left[\left(C_{t}^{*}, S_{t}^{*}\right)\right]=(0,0)$, both $\theta$ and $\bar{\nu}$ are undetermined, whereas $\bar{u}=0$.

The determinant of the Hessian matrix of $K_{C_{t}^{*}, S_{t}^{*}}$ at $(u \cos \nu, u \sin \nu)$ takes the compact form (11). This expression is obtained with the help of computer algebra. As expected, this determinant does not depend on $\nu$. From (6) follows $\lim _{u \rightarrow 0} A^{\prime}(u)=1-\lim _{u \rightarrow 0} A(u) / u$. Thus, from l'Hôpital rule,

$$
\lim _{u \rightarrow 0} \frac{A(u)}{u}=\lim _{u \rightarrow 0} A^{\prime}(u)=\frac{1}{2}
$$


This allows to compute (12). Note also that if $N_{t}=n$ a.s., for some $n \in \mathbb{N} \backslash\{0\}$, then (11) and (12) do indeed simplify to (4): precisely, $\sigma_{t}^{2}(u)=n^{2} \sigma^{2}(u), \forall u>0$ and as $u \rightarrow 0$. Differentiating the first equality in (14) with respect to $r$ leads to

$$
\frac{\mathrm{d}}{\mathrm{d} r} \bar{u}=\frac{A(\bar{u})}{\bar{u}} \frac{K_{N_{t}^{0}}^{\prime}\left(\log I_{0}(\bar{u})\right)}{\sigma_{t}^{2}(\bar{u})}>0, \quad \forall r>0 .
$$

Thus the saddlepoint $\bar{u}$ is an increasing function of $r$.

We can now write the saddlepoint approximation to $h_{t}^{*}(c, s)$, the conditional density of $\left(C_{t}^{*}, S_{t}^{*}\right)$ given $\left\{N_{t}>0\right\}$ at $(c, s)=r(\cos \theta, \sin \theta) \in \mathbb{R}^{2} \backslash\{(0,0)\}$, as

$$
\begin{aligned}
\tilde{h}_{t}^{*}(c, s) & =\left\{2 \pi \sigma_{t}(\bar{u})\right\}^{-1} \exp \left\{K_{C_{t}^{*}, S_{t}^{*}}(\bar{u} \cos \bar{\nu}, \bar{u} \sin \bar{\nu})-\bar{u} \cos \bar{\nu} r \cos \theta-\bar{u} \sin \bar{\nu} r \sin \theta\right\} \\
& =\left\{2 \pi \sigma_{t}(\bar{u})\right\}^{-1} M_{N_{t}^{0}}\left(\log I_{0}(\bar{u})\right) \mathrm{e}^{-\bar{u} r} .
\end{aligned}
$$

Also, the saddlepoint approximation to the defective density of $\left(C_{t}^{*}, S_{t}^{*}\right)$ at $(c, s) \in \mathbb{R}^{2} \backslash\{(0,0)\}$ is given by

$$
\tilde{h}_{t}^{*}(c, s)\left\{1-p_{0}(t)\right\}
$$

In terms of polar coordinates, the saddlepoint approximation to $g_{t}^{*}(r, \theta)$, the conditional density at $r>0$ and $\theta \in[0,2 \pi)$ of $\left(R_{t}^{*}, \mu_{t}^{*}\right)$ given $\left\{N_{t}>0\right\}$, is given by

$$
\tilde{g}_{t}^{*}(r, \theta)=\left\{2 \pi \sigma_{t}(\bar{u})\right\}^{-1} M_{N_{t}^{0}}\left(\log I_{0}(\bar{u})\right) r \mathrm{e}^{-\bar{u} r},
$$

whereas the saddlepoint approximation to the defective density of $\left(R_{t}^{*}, \mu_{t}^{*}\right)$ at $r>0$ and $\theta \in[0,2 \pi)$ is given by

$$
\tilde{g}_{t}^{*}(r, \theta)\left\{1-p_{0}(t)\right\}
$$

The saddlepoint approximation (16) and the Second factorization lemma conclude the proof.

\subsection{Von Mises case}

Assume now the $\operatorname{vM}(\mu, \kappa)$ distribution with density (1) for $\theta_{1}, \theta_{2}, \ldots$, where $\mu \in[0,2 \pi)$ and $\kappa \geq 0$. Denote by $h_{\mu, \kappa, t}^{*}, g_{\mu, \kappa, t}^{*}$ and $q_{\kappa, t}^{*}$ the conditional densities of $\left(C_{t}^{*}, S_{t}^{*}\right),\left(R_{t}^{*}, \mu_{t}^{*}\right)$ and $R_{t}^{*}$ given $\left\{N_{t}>0\right\}$, under the $\operatorname{vM}(\mu, \kappa)$ distribution. Note that $h_{\mu, 0, t}^{*}=h_{t}^{*}, g_{\mu, 0, t}^{*}=g_{t}^{*}(\mu$ being irrelevant) and $q_{0, t}^{*}=q_{t}^{*}$. Denote by $\mathrm{P}_{\mu, \kappa}$ the probability measure over $(\Omega, \mathcal{F})$, under the $\operatorname{vM}(\mu, \kappa)$ distribution, and denote by $\mathrm{E}_{\mu, \kappa}$ the associated expectation functional.

Lemma 3.3 (Third factorization lemma). $\forall r>0, \theta \in[0,2 \pi)$,

$$
g_{\mu, \kappa, t}^{*}(r, \theta)=f(\theta \mid \mu, r \kappa) q_{\kappa, t}^{*}(r) .
$$


Proof Define also $h_{\mu, \kappa, n}$ and $g_{\mu, \kappa, n}$ as the densities of $\left(C_{n}, S_{n}\right)$ and $\left(R_{n}, \mu_{n}\right)$ under the $\operatorname{vM}(\mu, \kappa)$ distribution. Thus $h_{\mu, 0, n}=h_{n}$ and $g_{\mu, 0, n}=g_{n}$ ( $\mu$ being irrelevant). Let $r>0, \theta \in$ $[0,2 \pi), c=r \cos \theta$ and $s=r \sin \theta$. Then, using the First factorization theorem, we have

$$
\begin{aligned}
& g_{\mu, \kappa, t}^{*}(r, \theta) \mathrm{d} r \mathrm{~d} \theta=\mathrm{P}_{\mu, \kappa}\left[R_{t}^{*} \in(r, r+\mathrm{d} r), \mu_{t}^{*} \in(\theta, \theta+\mathrm{d} \theta) \mid N_{t}>0\right] \\
& =\sum_{n=0}^{\infty} \mathrm{P}_{\mu, \kappa}\left[R_{t}^{*} \in(r, r+\mathrm{d} r), \mu_{t}^{*} \in(\theta, \theta+\mathrm{d} \theta), N_{t}=n \mid N_{t}>0\right] \\
& =\sum_{n=1}^{\infty} g_{\mu, \kappa, n}(r, \theta) \mathrm{d} r \mathrm{~d} \theta p_{n}^{0}(t) \\
& =\sum_{n=1}^{\infty} h_{\mu, \kappa, n}(r \cos \theta, r \sin \theta) r \mathrm{~d} r \mathrm{~d} \theta p_{n}^{0}(t) \\
& =\sum_{n=1}^{\infty} h_{\mu, \kappa, n}(c, s) \mathrm{d} c \mathrm{~d} s p_{n}^{0}(t) \\
& =\sum_{n=1}^{\infty} \int \ldots \int_{D(c, s)} \prod_{j=1}^{n} f\left(\theta_{j} \mid \mu, \kappa\right) \mathrm{d} \theta_{1} \ldots \mathrm{d} \theta_{n} p_{n}^{0}(t) \\
& =\sum_{n=1}^{\infty} I_{0}^{-n}(\kappa) \exp \{\kappa \cos \mu c+\kappa \sin \mu s\} h_{\mu, 0, n}(c, s) \mathrm{d} c \mathrm{~d} s p_{n}^{0}(t) \\
& =\sum_{n=1}^{\infty} I_{0}^{-n}(\kappa) \exp \{\kappa \cos \mu r \cos \theta+\kappa \sin \mu r \sin \theta\} \\
& h_{n}(r \cos \theta, r \sin \theta) r \mathrm{~d} r \mathrm{~d} \theta p_{n}^{0}(t) \\
& =\exp \{\kappa r \cos (\theta-\mu)\} \sum_{n=1}^{\infty} I_{0}^{-n}(\kappa) g_{n}(r, \theta) \mathrm{d} r \mathrm{~d} \theta p_{n}^{0}(t) \\
& =\frac{1}{2 \pi I_{0}(r \kappa)} \exp \{\kappa r \cos (\theta-\mu)\} \sum_{n=1}^{\infty} \frac{I_{0}(r \kappa)}{I_{0}^{n}(\kappa)} q_{n}(r) p_{n}^{0}(t) \mathrm{d} r \mathrm{~d} \theta,
\end{aligned}
$$

where $D(c, s)=\left\{\left(\theta_{1}, \ldots, \theta_{n}\right) \in[0,2 \pi)^{n} \mid \sum_{j=1}^{n} \cos \theta_{j} \in(c, c+\mathrm{d} c), \sum_{j=1}^{n} \sin \theta_{j} \in(s, s+\mathrm{d} s)\right\}$.

By integrating with respect to $\theta$ the last formula of the above proof and by using the Tilting lemma, we obtain

$$
\begin{aligned}
q_{\kappa, t}^{*}(r) & =\sum_{n=1}^{\infty} \frac{I_{0}(r \kappa)}{I_{0}^{n}(\kappa)} q_{n}(r) p_{n}^{0}(t) \\
& =\sum_{n=1}^{\infty} q_{\kappa, n}(r) p_{n}^{0}(t),
\end{aligned}
$$

which does not depend on $\mu$. The major result of the article is the following.

Proposition 3.4. The saddlepoint approximation to $q_{\kappa, t}^{*}(r)$, the conditional density of $R_{t}^{*}$ given $\left\{N_{t}>0\right\}$, under the $\operatorname{vM}(\mu, \kappa)$ distribution, is given by

$$
\tilde{q}_{\kappa, t}^{*}(r)=\frac{I_{0}(\kappa r)}{\sigma_{\kappa, t}(\bar{l})} M_{N_{t}^{0}}(B(\bar{l}, \kappa)) r \mathrm{e}^{-\bar{l} r}
$$


the saddlepoint approximation to the survival function $\bar{Q}_{\kappa, t}^{*}(r)=\mathrm{P}_{\mu, \kappa}\left[R_{t}^{*}>r \mid N_{t}>0\right]$ is given by

$$
\tilde{\bar{Q}}_{\kappa, t}^{*}(r)=I_{0}(\kappa r) \int_{\bar{l}}^{\infty} \sigma_{\kappa, t}(l) M_{N_{t}^{0}}(B(l, \kappa)) \exp \left\{-l K_{N_{t}^{0}}^{\prime}(B(l, \kappa)) A(l)\right\} l \mathrm{~d} l,
$$

whereas the saddlepoint approximation to the defective density of $R_{t}^{*}$ is given by

$$
\tilde{q}_{\kappa, t}^{*}(r)\left\{1-p_{0}(t)\right\}
$$

where $\bar{l}>0$ is the solution in $l$ of

$$
K_{N_{t}^{0}}^{\prime}(B(l, \kappa)) A(l)-r=0
$$

$\forall r>0$, and where $\sigma_{\kappa, t}(\bar{l})$ is given by the formula

$$
\sigma_{\kappa, t}^{2}(l)=\frac{A(l)}{l} K_{N_{t}^{0}}^{\prime}(B(l, \kappa))\left\{K_{N_{t}^{0}}^{\prime}(B(l, \kappa)) A^{\prime}(l)+K_{N_{t}^{0}}^{\prime \prime}(B(l, \kappa)) A^{2}(l)\right\}
$$

where $A^{\prime}$ is evaluated by (6) and $l=l(u, \nu, \kappa, \mu)>0$, and by the formula

$$
\lim _{l \rightarrow 0} \sigma_{\kappa, t}^{2}(l)=\frac{1}{4} K_{N_{t}^{0}}^{\prime}\left(-\log I_{0}(\kappa)\right) .
$$

Let $c_{\kappa, t}^{*-1}=\int_{0}^{\infty} \tilde{q}_{\kappa, t}^{*}(r) \mathrm{d} r$, then $c_{\kappa, t}^{*} \tilde{q}_{\kappa, t}^{*}(r)$ is the normalized saddlepoint approximation to $q_{\kappa, t}^{*}(r), \forall r>0$.

Also, $\bar{l}$ is a continuous increasing function of $r$ over $(0, \infty), \bar{l} \rightarrow 0$, as $r \rightarrow 0$, and $\bar{l}=\kappa$ at $r=A(\kappa) \mathrm{E}\left[N_{t} \mid N_{t}>0\right]$.

Proof By using (5), we obtain the m.g.f. of $\left(C_{1}, S_{1}\right)$ under the $\operatorname{vM}(\mu, \kappa)$ distribution as

$$
\begin{aligned}
M_{\mu, \kappa}\left(v_{1}, v_{2}\right) & =\mathrm{E}_{\mu, \kappa}\left[\exp \left\{v_{1} C_{1}+v_{2} S_{1}\right\}\right] \\
& =\frac{1}{2 \pi I_{0}(\kappa)} \int_{0}^{2 \pi} \exp \{u \cos \nu \cos \theta+u \sin \nu \sin \theta+\kappa \cos \mu \cos \theta+\kappa \sin \mu \sin \theta\} \mathrm{d} \theta \\
& =\frac{M_{1}(u \cos \nu+\kappa \cos \mu, u \sin \nu+\kappa \sin \mu)}{I_{0}(\kappa)} \\
& =\frac{I_{0}\left(\left\{u^{2}+\kappa^{2}+2 u \kappa \cos (\nu-\mu)\right\}^{\frac{1}{2}}\right)}{I_{0}(\kappa)}
\end{aligned}
$$

where $\left(v_{1}, v_{2}\right)=u(\cos \nu, \sin \nu) \in \mathbb{R}^{2}$. Clearly $M_{\mu, 0}=M$, with $\mu$ irrelevant. Define the c.g.f. $K_{\mu, \kappa}=\log M_{\mu, \kappa}$. Following the same steps as in (13) however using (20), we obtain the m.g.f. of $\left(C_{t}^{*}, S_{t}^{*}\right)$ conditional on $\left\{N_{t}>0\right\}$, under the $\operatorname{vM}(\mu, \kappa)$ distribution, as

$$
\begin{aligned}
M_{\mu, \kappa, C_{t}^{*}, S_{t}^{*}}\left(v_{1}, v_{2}\right) & =\mathrm{E}_{\mu, \kappa}\left[\exp \left\{v_{1} C_{t}^{*}+v_{2} S_{t}^{*}\right\} \mid N_{t}>0\right]=M_{N_{t}^{0}}\left(\log M_{\mu, \kappa}\left(v_{1}, v_{2}\right)\right) \\
& =M_{N_{t}^{0}}\left(\log \frac{I_{0}\left(\left\{u^{2}+\kappa^{2}+2 u \kappa \cos (\nu-\mu)\right\}^{\frac{1}{2}}\right)}{I_{0}(\kappa)}\right)
\end{aligned}
$$


The saddlepoint at $(c, s)=r(\cos \theta, \sin \theta) \in \mathbb{R}^{2} \backslash\{(0,0)\}$ is the solution in $\left(v_{1}, v_{2}\right)=$ $u(\cos \nu, \sin \nu)$ of

$$
\left(\begin{array}{c}
\frac{\partial}{\partial v_{1}} K_{\mu, \kappa, C_{t}^{*}, S_{t}^{*}}\left(v_{1}, v_{2}\right) \\
\frac{\partial}{\partial v_{2}} K_{\mu, \kappa, C_{t}^{*}, S_{t}^{*}}\left(v_{1}, v_{2}\right)
\end{array}\right)=\left(\begin{array}{c}
c \\
s
\end{array}\right)
$$

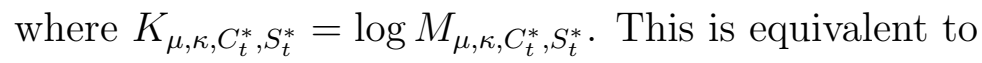

$$
Z_{\kappa, t}\left(\left\{u^{2}+\kappa^{2}+2 u \kappa \cos (\nu-\mu)\right\}^{\frac{1}{2}}\right)\left(\begin{array}{c}
u \cos \nu+\kappa \cos \mu \\
u \sin \nu+\kappa \sin \mu
\end{array}\right)=r\left(\begin{array}{c}
\cos \theta \\
\sin \theta
\end{array}\right)
$$

where we define

$$
Z_{\kappa, t}(l)=K_{N_{t}^{0}}^{\prime}(B(l, \kappa)) \frac{A(l)}{l}, \forall l>0,
$$

for $B(l, \kappa)$ given in (10). The saddlepoint equation (21) has an unique solution, denoted $(\bar{u}, \bar{\nu})$. In order to obtain interpretations of $(21)$, define by $l(u, \nu, \kappa, \mu)$ the length and by $\lambda(u, \nu, \kappa, \mu)$ the argument of the resultant vector $u(\cos \nu, \sin \nu)+\kappa(\cos \mu, \sin \mu)$. Thus

$$
l(u, \nu, \kappa, \mu)=\left\{u^{2}+\kappa^{2}+2 u \kappa \cos (\nu-\mu)\right\}^{\frac{1}{2}} .
$$

We first note that $l(u, \nu, \kappa, \mu)=0$ and $\lambda(u, \nu, \kappa, \mu)$ is undetermined iff $u=\kappa$ and $\nu=$ $(\mu+\pi) \bmod 2 \pi$. Next, $(21)$ can be re-expressed as

$$
Z_{\kappa, t}(l(u, \nu, \kappa, \mu)) l(u, \nu, \kappa, \mu)=r \text { and } \lambda(u, \nu, \kappa, \mu)=\theta .
$$

It is then clear that we cannot separate the variables $u$ and $\nu$ (that is, obtain one equation with $u$ only and another one in $\nu$ only) as was the case with (14), unless $\kappa=0$. However, (23) provides two independent equations in $\lambda$ and $l$, where the second equation is the identity. Under this reparametrization, these equations have same nature as the saddlepoint equations (14), obtained under isotropy.

The determinant of the Hessian matrix of $K_{\mu, \kappa, C_{t}^{*}, S_{t}^{*}}$ at $(u \cos \nu, u \sin \nu)$ can be obtained by computer algebra in the form of (18), where $l=l(u, \nu, \kappa, \mu)>0$. Using (15) we obtain the limit (19). If $\kappa=0$, then (18) simplifies to (11): precisely, $\sigma_{0, t}^{2}(l(u, \nu, 0, \mu))=\sigma_{t}^{2}(u)$. Note also that if $N_{t}=n$ a.s., for some $n \in \mathbb{N} \backslash\{0\}$, then $K_{N_{t}^{0}}^{\prime}(B(l, \kappa))=n$ and $K_{N_{t}^{0}}^{\prime \prime}(B(l, \kappa))=0$, which in turn implies that (18) simplifies to (4): $\sigma_{\kappa, t}^{2}(l)=n^{2} \sigma^{2}(l), \forall l>0$ and as $l \rightarrow 0$.

The saddlepoint approximation to $h_{\mu, \kappa, t}^{*}(c, s)$, the conditional density of $\left(C_{t}^{*}, S_{t}^{*}\right)$ given $\left\{N_{t}>0\right\}$ at $(c, s) \in \mathbb{R}^{2} \backslash\{(0,0)\}$, is given by

$$
\begin{aligned}
\tilde{h}_{\mu, \kappa, t}^{*}(c, s)= & \left\{2 \pi \sigma_{\kappa, t}(l(\bar{u}, \bar{\nu}, \kappa, \mu))\right\}^{-1} \\
& \cdot \exp \left\{K_{\mu, \kappa, C_{t}^{*}, S_{t}^{*}}(\bar{u} \cos \bar{\nu}, \bar{u} \sin \bar{\nu})-\bar{u} \cos \bar{\nu} r \cos \theta-\bar{u} \sin \bar{\nu} r \sin \theta\right\} \\
= & \left\{2 \pi \sigma_{\kappa, t}(l(\bar{u}, \bar{\nu}, \kappa, \mu))\right\}^{-1} M_{N_{t}^{0}}(B(l(\bar{u}, \bar{\nu}, \kappa, \mu), \kappa)) \exp \{-\cos (\theta-\bar{\nu}) \bar{u} r\}
\end{aligned}
$$

and the approximation to the defective density of $\left(C_{t}^{*}, S_{t}^{*}\right)$ at $(c, s) \in \mathbb{R}^{2} \backslash\{(0,0)\}$ is given by

$$
\tilde{h}_{\mu, \kappa, t}^{*}(c, s)\left\{1-p_{0}(t)\right\} .
$$


Thus the saddlepoint approximation to $g_{\mu, \kappa, t}^{*}(r, \theta)$, the conditional density of $\left(R_{t}^{*}, \mu_{t}^{*}\right)$ given $\left\{N_{t}>0\right\}$ at $(r, \theta) \in(0, \infty) \times[0,2 \pi)$, is given by

$$
\tilde{g}_{\mu, \kappa, t}^{*}(r, \theta)=\left\{2 \pi \sigma_{\kappa, t}(l(\bar{u}, \bar{\nu}, \kappa, \mu))\right\}^{-1} M_{N_{t}^{0}}(B(l(\bar{u}, \bar{\nu}, \kappa, \mu), \kappa)) r \exp \{-\cos (\theta-\bar{\nu}) \bar{u} r\},
$$

whereas the saddlepoint approximation to the defective density of $\left(R_{t}^{*}, \mu_{t}^{*}\right)$ at this point is given by

$$
\tilde{g}_{\mu, \kappa, t}^{*}(r, \theta)\left\{1-p_{0}(t)\right\} .
$$

The Third factorization lemma and (24) lead to

$$
\tilde{q}_{\kappa, t}^{*}(r)=\frac{I_{0}(\kappa r)}{\sigma_{\kappa, t}(l(\bar{u}, \bar{\nu}, \kappa, \mu))} M_{N_{t}}(B(l(\bar{u}, \bar{\nu}, \kappa, \mu), \kappa)) r \exp \{-r[\bar{u} \cos (\theta-\bar{\nu})+\kappa \cos (\theta-\mu)]\} .
$$

The above exponent can be re-expressed as

$$
\begin{aligned}
-r[(\bar{u} \cos & \bar{\nu}+\kappa \cos \mu) \cos \theta+(\bar{u} \sin \bar{\nu}+\kappa \sin \mu) \sin \theta]= \\
& -Z_{\kappa, t}\left(\left\{\bar{u}^{2}+\kappa^{2}+2 \bar{u} \kappa \cos (\bar{\nu}-\mu)\right\}^{\frac{1}{2}}\right)\left\{(\bar{u} \cos \bar{\nu}+\kappa \cos \mu)^{2}+(\bar{u} \sin \bar{\nu}+\kappa \sin \mu)^{2}\right\}= \\
& -Z_{\kappa, t}\left(\left\{\bar{u}^{2}+\kappa^{2}+2 \bar{u} \kappa \cos (\bar{\nu}-\mu)\right\}^{\frac{1}{2}}\right)\left\{\bar{u}^{2}+\kappa^{2}+2 \bar{u} \kappa \cos (\bar{\nu}-\mu)\right\}= \\
& -Z_{\kappa, t}(l(\bar{u}, \bar{\nu}, \kappa, \mu)) l^{2}(\bar{u}, \bar{\nu}, \kappa, \mu)= \\
& -r l(\bar{u}, \bar{\nu}, \kappa, \mu),
\end{aligned}
$$

by using equations (21), (22) and (23). Thus (25) depends on $\kappa$ and $l(\bar{u}, \bar{\nu}, \kappa, \mu)$ only. As it does not depend on $\lambda(\bar{u}, \bar{\nu}, \kappa, \mu)$, the second equation of (23) tells that the saddlepoint approximation (25) does not depend on $\theta$ either. Further, (25) depends on $\mu$ only through $l(\bar{u}, \bar{\nu}, \kappa, \mu)$, so from (22) only through $\bar{\nu}-\mu$ : any variation of $\mu$ is canceled by the same variation of $\bar{\nu}$. In other terms, the value of $\mu$ is irrelevant and one can simply set $\mu=0$, without loss of generality. But even the particular values of $(\bar{u}, \bar{\nu})$ are irrelevant, as long as they lead to the desired value $l(\bar{u}, \bar{\nu}, \kappa, 0)$, which is obtained from the first equation in (23), i.e. from (17), and denoted $\bar{l}$. Thus the approximations to the conditional and defective densities and hold.

The given approximation to the survival function is directly obtained by integration of the saddlepoint approximation to the density, after a practical change variable of integration which avoids the necessity of computing the saddlepoint at each ordinate. Precisely, we have

$$
\begin{aligned}
\tilde{\bar{Q}}_{\kappa, t}^{*}(r) & =\int_{r}^{\infty} \tilde{q}_{\kappa, t}^{*}(x) \mathrm{d} x \\
& =I_{0}(\kappa r) \int_{r}^{\infty} \sigma_{\kappa, t}^{-1}\left(\bar{l}_{x}\right) M_{N_{t}^{0}}\left(B\left(\bar{l}_{x}, \kappa\right)\right) x \exp \left\{-\bar{l}_{x} x\right\} \mathrm{d} x \\
& =I_{0}(\kappa r) \int_{\bar{l}}^{\infty} \sigma_{\kappa, t}^{-1}(l) M_{N_{t}^{0}}(B(l, \kappa)) x(l) \exp \{-l x(l)\} \frac{\mathrm{d}}{\mathrm{d} l} x(l) \mathrm{d} l,
\end{aligned}
$$

where $\bar{l}_{x}$ is the solution in $l$ of (17) with $x$ replacing $r$ and where $x(l)=K_{N_{t}^{0}}^{\prime}(B(l, \kappa)) A(l)$. Inserting

$$
\frac{\mathrm{d}}{\mathrm{d} l} x(l)=\frac{l}{A(l)} \frac{\sigma_{\kappa, t}^{2}(l)}{K_{N_{t}^{0}}^{\prime}(B(l, \kappa))}
$$


in the last integral leads to the claimed approximation to the survival function.

The positivity of $\mathrm{d} x(l) / \mathrm{d} l$ implies that $\bar{l}$ increases with $x$, i.e. $\bar{l}$ increases with $r$. At the point of conditional expectation of $\left(C_{t}^{*}, S_{t}^{*}\right)$, we must have $\bar{u}=0$. This expectation is given by

$$
\begin{aligned}
\mathrm{E}_{\mu, \kappa}\left[\left(\begin{array}{c}
C_{t}^{*} \\
S_{t}^{*}
\end{array}\right) \mid N_{t}>0\right] & =\left.\left(\begin{array}{c}
\frac{\partial}{\partial v_{1}} K_{\mu, \kappa, C_{t}^{*}, S_{t}^{*}}\left(v_{1}, v_{2}\right) \\
\frac{\partial}{\partial v_{2}} K_{\mu, \kappa, C_{t}^{*}, S_{t}^{*}}\left(v_{1}, v_{2}\right)
\end{array}\right)\right|_{v_{1}=v_{2}=0} \\
& =K_{N_{t}^{\prime}}^{\prime}(B(\kappa, \kappa)) A(\kappa)\left(\begin{array}{c}
\cos \mu \\
\sin \mu
\end{array}\right) \\
& =A(\kappa) \mathrm{E}\left[N_{t} \mid N_{t}>0\right]\left(\begin{array}{c}
\cos \mu \\
\sin \mu
\end{array}\right) .
\end{aligned}
$$

From (22) follows that at this expectation $l(0, \nu, \kappa, \mu)=\kappa, \forall \nu \in[0,2 \pi)$. Thus $\bar{l}=\kappa$ is the saddlepoint for the saddlepoint approximation to the conditional density of $R_{t}^{*}$ given $\left\{N_{t}>0\right\}$ at point

$$
r=\left\|\mathrm{E}_{\mu, \kappa}\left[\left(\begin{array}{c}
C_{t}^{*} \\
S_{t}^{*}
\end{array}\right) \mid N_{t}>0\right]\right\|=A(\kappa) \mathrm{E}\left[N_{t} \mid N_{t}>0\right] .
$$

Moreover (23) tells that $\bar{l}=l(\bar{u}, \bar{\nu}, \kappa, \mu) \rightarrow 0$, as $r \rightarrow 0$.

An important feature of this saddlepoint approximation is that, although the resultant is two-dimensional, the saddlepoint equation (17) is one-dimensional and thus easy to obtain. Some further remarks are the following. The approximation $\tilde{q}_{\kappa, t}^{*}$ does not depend on $\mu$. If $N_{t}=n$ a.s., for some $n \in \mathbb{N} \backslash\{0\}$, then $M_{N_{t}^{0}}(B(l, \kappa))=\mathrm{e}^{n B(l, \kappa)}$ and Proposition 3.4 simplifies to Proposition 2.6. Note also that the proposed approximation to the survival function requires numerical integration, which is however simple to perform, because of the smoothness of the integrand.

\section{Compounding with inhomogeneous birth processes}

This section provides the major applications of the saddlepoint approximations derived in Section 3 for general counting processes. We consider a Markovian counting process $\left\{N_{t}\right\}_{t \geq 0}$ with transition probabilities given by

$$
p_{k, k+n}(s, t)=\mathrm{P}\left[N_{t}-N_{s}=n \mid N_{s}=k\right], \quad \forall s, t \geq 0 \text { such that } s \leq t \text { and } k, n \in \mathbb{N} \text {. }
$$

Then $\left\{N_{t}\right\}_{t \geq 0}$ is an inhomogeneous birth process if, $\forall t>0$ and $k \in \mathbb{N}$,

$$
p_{k, k+n}(t, t+h)= \begin{cases}1-\lambda_{k}(t) h+\mathrm{o}(h), & \text { if } n=0, \\ \lambda_{k}(t) h+\mathrm{o}(h), & \text { if } n=1, \\ \mathrm{o}(h), & \text { if } n=2,3, \ldots,\end{cases}
$$


as $h \rightarrow 0, h>0$, where $\lambda_{k}:[0, \infty) \rightarrow[0, \infty]$, for $k \in \mathbb{N}$, are the transition intensity functions. It is assumed that the transition intensity functions are continuous in $t$ on $(0, \infty)$. It is allowed that $\lambda_{k}(0)=\infty$, for some $k \in \mathbb{N}$, however $\int_{0}^{t} \lambda_{0}(s) \mathrm{d} s<\infty, \forall t \in(0, \infty)$, is required. This insures that $N_{0}=0$ a.s., as assumed at the beginning of Section 3.1. It is quite direct to understand that a birth process is determined by its transition intensity functions. The other way around, any set of nonnegative functions $\lambda_{k}$, for $k \in \mathbb{N}$, continuous over $(0, \infty)$ and satisfying

$$
\sum_{k=n}^{\infty}\left(\max _{0 \leq s<t} \lambda_{k}(s)\right)^{-1}=\infty, \forall t>0, n \in \mathbb{N}
$$

is the set of transition intensity functions of some birth process. As the sum appearing in the above condition grows with $t$ and $n$, one should really understand that the divergence to infinity is required for $t$ and $n$ arbitrarily large. This condition limits the growth of the transition intensity functions. The reference for these conditions is p. 59-60 of Grandell (1997).

If the transition intensity functions do not depend on their argument $t$, then $\left\{N_{t}\right\}_{t \geq 0}$ is an homogeneous birth process, whereas if the transition intensity functions do not depend on their index $k$, then it is a birth process with independent increments. In the next two subsections we consider the three most important birth processes: the Poisson, the binomial and the negative binomial. A practical reference for birth processes is Section 6.6 of Klugman et al. (2008).

\subsection{Compound Poisson random walk}

Transition intensities of the form $\lambda_{k}(t)=\lambda(t), \forall t \geq 0$ and $k \in \mathbb{N}$, determine the Poisson process. The Poisson process possesses various practical properties, such as superposition and thinning. It is inhomogeneous, unless the transition intensity function is constant, and it has independent increments. The transition probabilities are given by

$$
p_{k, k+n}(s, t)=\exp \{-[\Lambda(t)-\Lambda(s)]\} \frac{[\Lambda(t)-\Lambda(s)]^{n}}{n !}
$$

where $\Lambda(t)=\int_{0}^{t} \lambda(s) \mathrm{d} s$ is the expectation function, $\forall s \leq t$ and $k, n \in \mathbb{N}$. Let $t \geq 0$.

Theorem 4.1. The saddlepoint approximation to $q_{\kappa, t}^{*}(r)$, the conditional density of the compound Poisson random walk given $\left\{N_{t}>0\right\}$ and under the $\operatorname{vM}(\mu, \kappa)$ distribution, is given by

$$
\tilde{q}_{\kappa, t}^{*}(r)=\frac{I_{0}(\kappa r)}{\sigma_{\kappa, t}(\bar{l})} \frac{\mathrm{e}^{U(\bar{l}, \kappa, t)}-1}{\mathrm{e}^{\Lambda(t)}-1} r \mathrm{e}^{-\bar{l} r},
$$

where $\sigma_{\kappa, t}$ is given by

$$
\sigma_{\kappa, t}^{2}(l)=\frac{A(l)}{l}\left(\frac{U(l, \kappa, t)}{1-\mathrm{e}^{-U(l, \kappa, t)}}\right)^{2}\left\{A^{\prime}(l)+\left[1-\frac{U(l, \kappa, t)}{\mathrm{e}^{U(l, \kappa, t)}-1}\right] A^{2}(l)\right\},
$$




$$
U(l, \kappa, t)=\Lambda(t) \frac{I_{0}(l)}{I_{0}(\kappa)}, \forall l>0,
$$

and where $\bar{l}>0$ is the solution in $l$ of

$$
\frac{U(l, \kappa, t)}{1-\mathrm{e}^{-U(l, \kappa, t)}} A(l)-r=0
$$

$\forall r>0$.

Proof We easily obtain $K_{N_{t}}(v)=\Lambda(t)\left(\mathrm{e}^{v}-1\right)$ and $K_{N_{t}^{0}}(v)=-\Lambda(t)-\log \left\{1-\mathrm{e}^{-\Lambda(t)}\right\}+$ $\log \left(\exp \left\{\Lambda(t) \mathrm{e}^{v}\right\}-1\right), \forall v \in \mathbb{R}$. With this c.g.f. and (18), we obtain (27) as well as

$$
Z_{\kappa, t}(l)=\frac{A(l)}{l} \frac{U(l, \kappa, t)}{1-\mathrm{e}^{-U(l, \kappa, t)}},
$$

where $U$ is given in (28). These two last formulae, (23) and Proposition 3.4 yield the theorem.

The survival function can be trivially obtained from Proposition 3.4.

An informal justification of the asymptotic validity of the saddlepoint approximation for large values of $t$ is the following. The expected number of steps is $\mathrm{E}\left[N_{t}\right]=\Lambda(t)$, which usually tends to infinity, as $t \rightarrow \infty$. (This happens e.g. with a periodic transition intensity function.) In this case the central limit theorem applies and the relative error of the saddlepoint approximation is expected to vanish asymptotically.

\subsection{Compound contagious random walk}

We now consider transition intensities with linear contagion, precisely of the form

$$
\lambda_{k}(t)=\alpha+\beta k
$$

$\forall t \geq 0$, where $\alpha \geq 0, \beta \neq 0$ satisfy $\alpha+\beta k \geq 0, \forall k \in \mathbb{N}$. This choice leads to homogeneous processes with dependent increments and it can be shown that the transition probabilities are

$$
p_{k, k+n}(s, t)=\left(\begin{array}{c}
\frac{\alpha}{\beta}+k+n-1 \\
n
\end{array}\right) \mathrm{e}^{-(\alpha+\beta k)(t-s)}\left(1-\mathrm{e}^{-\beta(t-s)}\right)^{n},
$$

$\forall s, t \geq 0$ such that $s \leq t$ and $k, n \in \mathbb{N}$. The case $\beta>0$ is presented in Section 4.2 .1 and the case $\beta<0$ is presented in Section 4.2.2.

\subsubsection{Compound negative binomial random walk}

When $\beta>0$, (29) yields the negative binomial transition probabilities

$$
p_{k, k+n}(s, t)=\left(\begin{array}{c}
\frac{\alpha}{\beta}+k+n-1 \\
n
\end{array}\right)\left(\mathrm{e}^{-\beta(t-s)}\right)^{\frac{\alpha}{\beta}+k}\left(1-\mathrm{e}^{-\beta(t-s)}\right)^{n},
$$

$\forall s, t \geq 0$ such that $s \leq t$ and $k, n \in \mathbb{N}$. This negative binomial process should be used when past occurrences indicate increase of future occurrences. Let $t \geq 0$. 
Theorem 4.2. The saddlepoint approximation to $q_{\kappa, t}^{*}(r)$, the conditional density of the negative binomial random walk given $\left\{N_{t}>0\right\}$ and under the $\operatorname{vM}(\mu, \kappa)$ distribution, is given by

$$
\tilde{q}_{\kappa, t}^{*}(r)=\frac{I_{0}(\kappa r)}{\sigma_{\kappa, t}(\bar{l})} V^{-\frac{\alpha}{\beta}}(\bar{l}, \kappa, t) \mathrm{e}^{-\alpha t} r \mathrm{e}^{-\bar{l} r}
$$

where $\sigma_{\kappa, t}$ is given by

$$
\begin{aligned}
& \sigma_{\kappa, t}^{2}(l)= \frac{A(l)}{l}\left(\frac{\alpha}{\beta} \frac{1-V(l, \kappa, t)}{V(l, \kappa, t)\left[1-V^{\frac{\alpha}{\beta}}(l, \kappa, t)\right]}\right)^{2} \\
& \cdot\left\{A^{\prime}(l)+A^{2}(l) \frac{1-\left\{1+\frac{\alpha}{\beta}[1-V(l, \kappa, t)]\right\} V^{\frac{\alpha}{\beta}}(l, \kappa, t)}{V(l, \kappa, t)\left[1-V^{\frac{\alpha}{\beta}}(l, \kappa, t)\right]}\right\}, \\
& V(l, \kappa, t)=1-\left(1-\mathrm{e}^{-\beta t}\right) \frac{I_{0}(l)}{I_{0}(\kappa)}, \forall l>0,
\end{aligned}
$$

and where $\bar{l}>0$ is the solution in $l$ of

$$
\frac{1-V(l, \kappa, t)}{V(l, \kappa, t)\left[1-V^{\frac{\alpha}{\beta}}(l, \kappa, t)\right]} A(l)-\frac{\beta}{\alpha} r=0,
$$

$\forall r>0$.

Proof We easily obtain $K_{N_{t}}(v)=-\alpha t-\alpha / \beta \quad \log \left\{1-\left(1-\mathrm{e}^{-\beta t}\right) \mathrm{e}^{v}\right\}$ and $K_{N_{t}^{0}}(v)=$ $-\alpha t-\log \left\{1-\mathrm{e}^{-\alpha t}\right\}+\log \left\{\left[1-\left(1-\mathrm{e}^{-\beta t}\right) \mathrm{e}^{v}\right]^{-\frac{\alpha}{\beta}}-1\right\}, \forall v<-\log \left\{1-\mathrm{e}^{-\beta t}\right\}$. With this c.g.f. and (18) we find (30) as well as

$$
Z_{\kappa, t}(l)=\frac{A(l)}{l} \frac{\alpha}{\beta} \frac{1-V(l, \kappa, t)}{V(l, \kappa, t)\left[1-V^{\frac{\alpha}{\beta}}(l, \kappa, t)\right]},
$$

where $V$ is given by (31). This with (23) and Proposition 3.4 lead to the theorem.

The asymptotic validity of the saddlepoint approximation for large values of $t$ could be informally explained as follows. The expected number of steps is $\mathrm{E}\left[N_{t}\right]=\alpha / \beta\left(e^{\beta t}-1\right) \rightarrow \infty$, as $t \rightarrow \infty$ and so the error of the saddlepoint approximations should vanish asymptotically.

\subsubsection{Compound binomial random walk}

Consider now $\beta<0$ and assume $-\alpha / \beta \in \mathbb{N} \backslash\{0\}$. With some algebra, we can re-express (29) in the form of binomial transition probabilities

$$
p_{k, k+n}(s, t)=\left(\begin{array}{c}
-\frac{\alpha}{\beta}-k \\
n
\end{array}\right)\left(\mathrm{e}^{\beta(t-s)}\right)^{-\frac{\alpha}{\beta}-k-n}\left(1-\mathrm{e}^{\beta(t-s)}\right)^{n},
$$

for $k=0, \ldots,-\alpha / \beta-1, n=0, \ldots,-\alpha / \beta-k$ and $\forall s \leq t$. If $k=-\alpha / \beta$, then $\lambda_{k}(t)=0$ and the binomial coefficient in (29) vanishes $\forall n \in \mathbb{N} \backslash\{0\}$. So at most $-\alpha / \beta$ events can 
occur. The binomial process is used when past events tend not to reappear, i.e. reappear less frequently. This happens when preventive steps against the causes are taken. Let $t \geq 0$. We easily obtain $K_{N_{t}}(v)=-\alpha t-\alpha / \beta \log \left\{1-\left(1-\mathrm{e}^{-\beta t}\right) \mathrm{e}^{v}\right\}, \forall v \in \mathbb{R}$. This c.g.f. has the same form as the one obtained for the negative binomial process, therefore Theorem 4.2 holds for the compound binomial random walk as well, when $\alpha$ and $\beta$ have the corresponding restrictions.

\section{Numerical illustration}

This section provides a numerical illustration of the accuracy of the saddlepoint approximation for the compound Poisson random walk of Section 4.1. The accuracy is determined through a comparison with the distribution obtained by Monte Carlo simulation, based on $5 \cdot 10^{5}$ generations. We study the homogeneous Poisson process with constant intensity or rate $\lambda=1$ at time $t=10$, where the steps are $\operatorname{vM}(0, \kappa)$ distributed, with $\kappa=0,1,2$ and 4 .

We consider the conditional densities of $R_{10}^{*}$ given $\left\{N_{10}>0\right\}$. The normalizing constants of the considered saddlepoint approximations to these densities are $c_{10}^{*-1}=0.960, c_{1,10}^{*-1}=$ $0.974, c_{2,10}^{*-1}=0.993$ and $c_{4,10}^{*-1}=1.005$. For the case $\kappa=0$, i.e. the isotropic case, Figure 1 shows that the normalized saddlepoint approximation provides a perfect fit of the histogram on the simulated values, indicating a very high accuracy of the saddlepoint approximation. For the case $\kappa=4$, Figure 2 shows that the normalized saddlepoint approximation is very accurate in this case too. We note the bumpy behavior of the left half of the histogram, which is however very well fitted and smoothed by the saddlepoint density. Finally, Figure 3 shows the normalized saddlepoint approximations to the conditional densities of $R_{10}^{*}$ given $\left\{N_{10}>0\right\}$ with $\kappa=0,1,2$ and 4 , appearing respectively from the left to the right. This figure shows the evolution of the densities with respect to $\kappa$. It is very fast to obtain with the saddlepoint approximation. Computing it by simulation would be substantially longer, also because it would require smoothing the histograms.

Note that the saddlepoint $\bar{l}$ is the fixed point in $l$ of the function $f(l)=A^{(-1)}(r /$ $\left.K_{N_{t}^{0}}^{\prime}(B(l, \kappa))\right)$, i.e. $\bar{l}=f(\bar{l})$. This function can be explicitly evaluated by using $(3)$ and so one may want to compute $\bar{l}$ through the fixed point iteration $l_{n+1}=f\left(l_{n}\right)$, for $n=0,1, \ldots$ However, this appears inappropriate for obtaining saddlepoints of abscissa values $r$ in upper tail of the density. This is due to the fact that arguments of $A^{(-1)}$ larger than one often appear during iterations. Moreover, $A$ becomes very flat over the right tail.

\section{Final remarks}

We conclude this article with some remarks. Computer algebra has been very helpful in this research work and all important algebraic computations have been done with Matlab. The numerical study has been performed with Matlab and the function fzero has been used for solving the saddlepoint equation. Matlab's programs used for these computations are available under http://www.stat.unibe.ch. 
Figure 1: Saddlepoint approximation to the conditional density of the compound Poisson random walk given $\left\{N_{10}>0\right\}$ with $\kappa=0$. The histogram shows the Monte Carlo estimation. The continuous/dashed line shows the saddlepoint approximation without/with normalization.

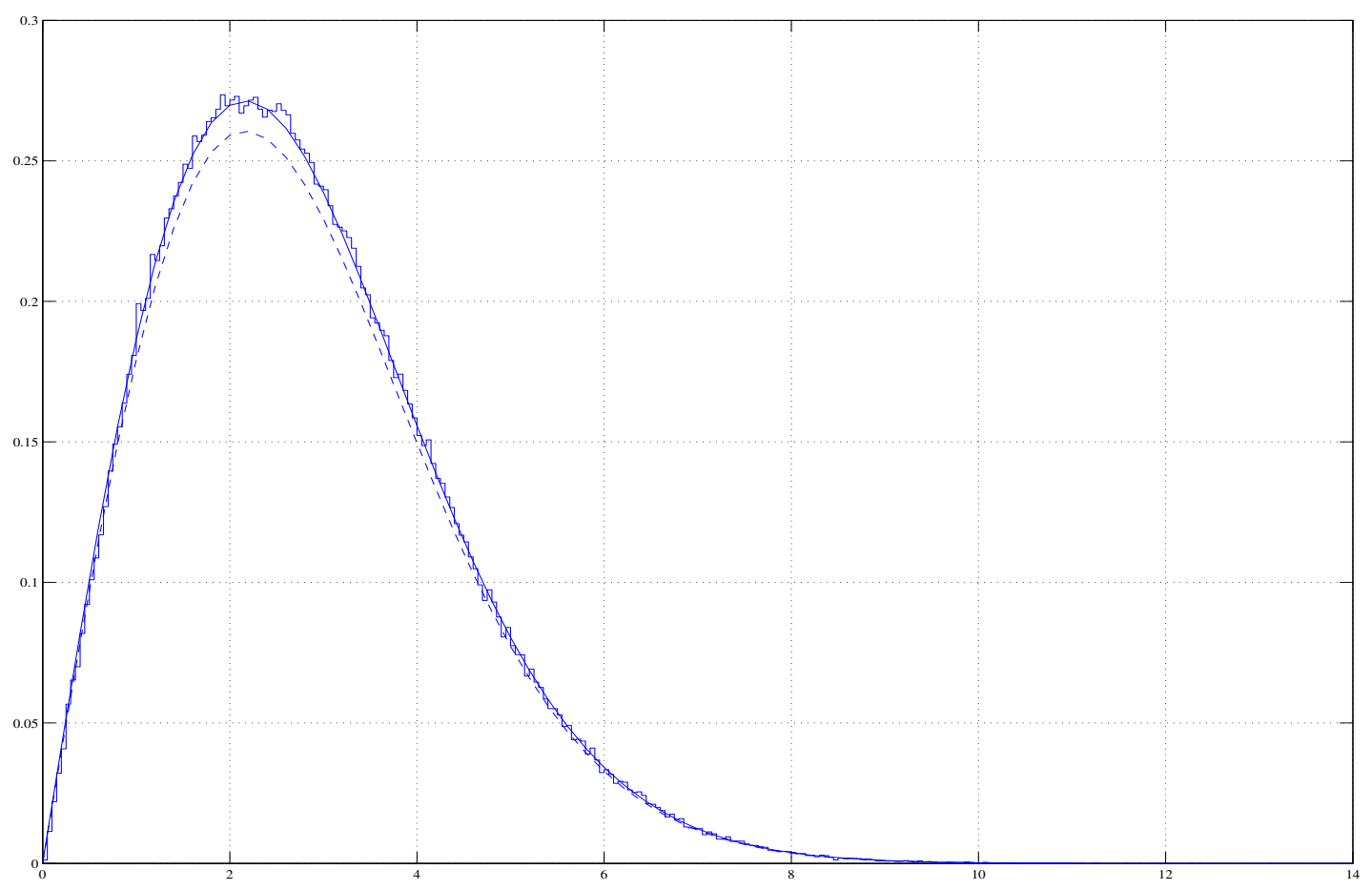


Figure 2: Saddlepoint approximation to the conditional density of the compound Poisson random walk given $\left\{N_{10}>0\right\}$ with $\kappa=4$. The histogram shows the Monte Carlo estimation. The continuous line shows the saddlepoint approximation with normalization.

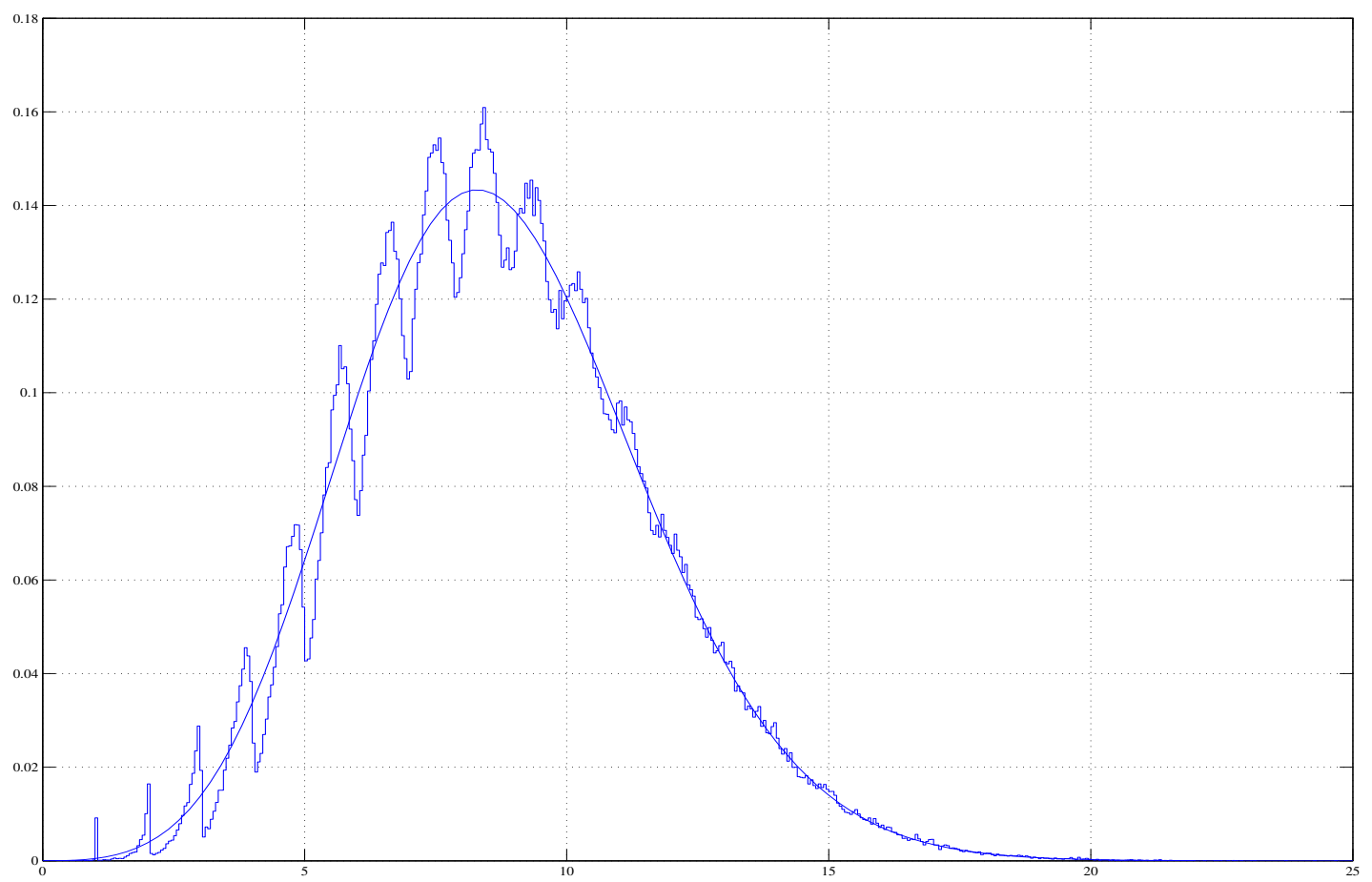


Figure 3: Saddlepoint approximations to the conditional density of the compound Poisson random walk given $\left\{N_{10}>0\right\}$ with normalization. The approximations with $\kappa=0,1,2$ and 4 appear respectively from the left to the right.

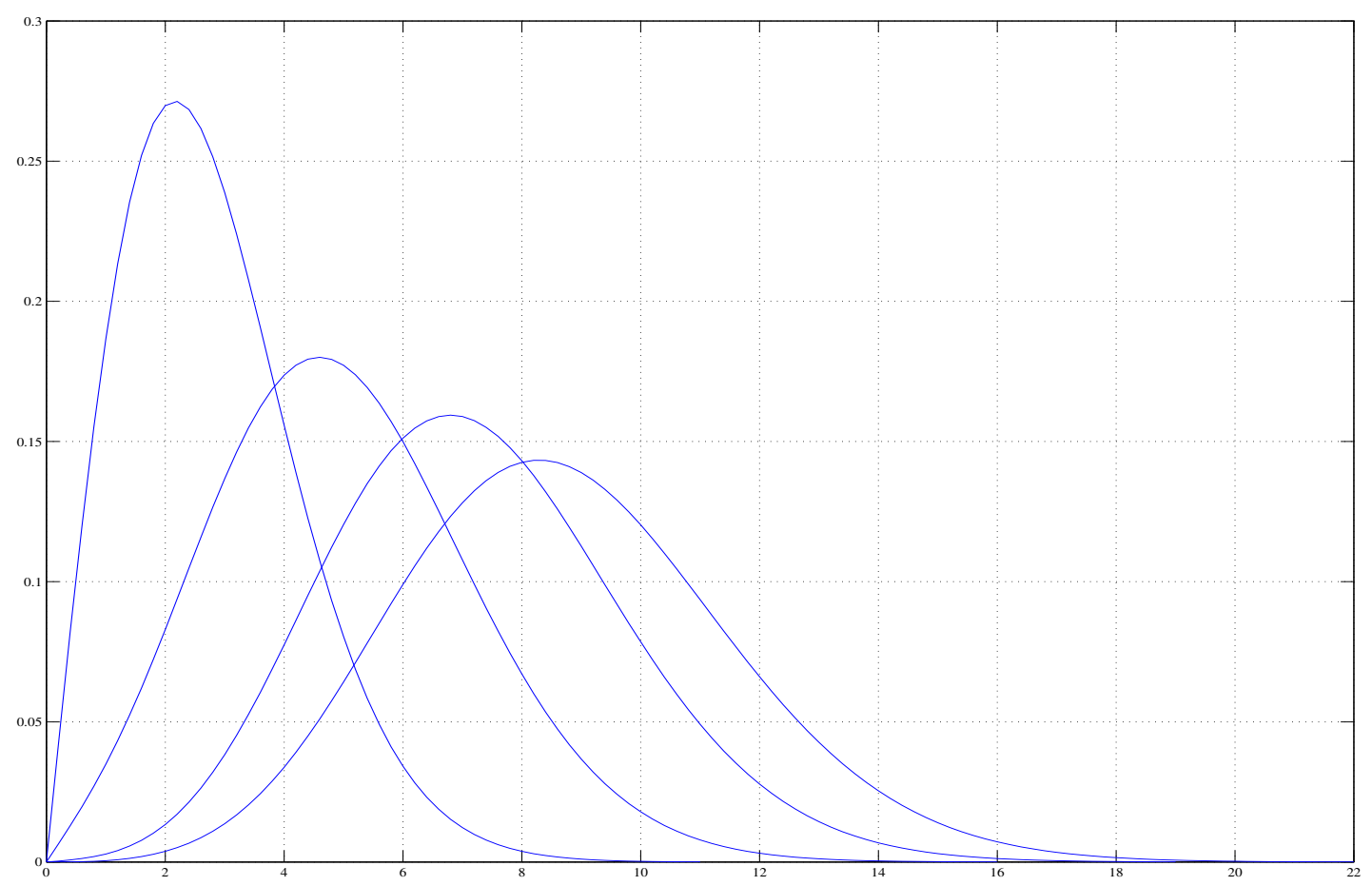


The saddlepoint approximation for any dimension $p>2$ but with a fixed number of summands only has been investigated in Gatto (2016). It should be possible to generalize the results of this article to any dimension $p>2$. With $p>2$, it may be convenient to replace the angular or polar representation of directions by the Cartesian. Also, the generalization of the determinants $\sigma_{t}^{2}(u)$ and $\sigma_{\kappa, t}^{2}(u)$ to $p>2$ may be laborious. Another open problem is the generalization of these saddlepoint approximations to the random flight with random step sizes of various distributions. Although Section 2.2 is limited to exponential lengths, it should provide the main ideas for this generalization. A further important problem is the generalization to non-identically distributed directions or step sizes.

\section{$7 \quad$ References}

Abramowitz, M., Stegun, I. E. (1972), Handbook of Mathematical Functions with Formulas, Graphs, and Mathematical Tables, Dover (reprint).

Barakat, R. (1973), "Isotropic random flights", Journal of Physics A: Mathematical, Nuclear and General, 6, 796-804.

Barber, M. N., Ninham, B. W. (1970), Random and Restricted Walks, Theory and Applications, Gordon and Breach.

Barndorff-Nielsen, O. E., Cox, D. R. (1989), Asymptotic Techniques for Use in Statistics, Chapman \& Hall.

Best, D. J., Fisher, N. I. (1981), "The bias of the maximum likelihood estimators of the von Mises-Fisher concentration parameters", Communications in Statistics, B, Simulation and Computation, 10, 493-502.

Daniels, H. E. (1954), "Saddlepoint approximations in statistics", The Annals of Mathematical Statistics, 25, 631-650.

Field, C. A., Ronchetti, E. (1990), Small Sample Asymptotics, Institute of Mathematical Statistics, 13 .

Field, C. A., Tingley, M. A. (1997), "Small sample asymptotics: applications in robustness", Handbook of Statistics, Elsevier, Volume 15, Chapter 18, 513-536.

Flory, P. J. (1969), Statistical Mechanics of Chain Molecules, Interscience.

Gatto, R. (2015), "Saddlepoint approximations", StatsRef: Statistics Reference Online, editors Balakrishnan et al., Wiley and Sons, 1-7.

DOI : 10.1002/9781118445112.stat01796.pub2

Gatto, R. (2016), Saddlepoint approximations to the distribution of the total distance of the multivariate isotropic and von Mises-Fisher random walks, technical report, Institute of Mathematical Statistics and Actuarial Science, University of Bern.

Gatto, R., Jammalamadaka, S. R. (2003). "Inference for wrapped symmetric $\alpha$-stable 
circular models", Sankhyā, A: Mathematical Statistics and Probability, 65, 2, 333-355.

Gatto, R., Mayer, M. (2005), "Saddlepoint approximations for some models of circular data", Statistical Methodology, 2, 233-248.

Grandell, J. (1997), Mixed Poisson Processes, Chapnan \& Hall.

Greenwood J. A., Durand D. (1955), "The distribution of length and components of the sum of $n$ random unit vectors", Annals of Mathematical Statistics, 26, 233-246.

Jammalamadaka, S. R., SenGupta, A. (2001), Topics in Circular Statistics, World Scientific Press.

Jensen, J. L. (1995a), Saddlepoint Approximations, Oxford University Press.

Jensen, J. L. (1995b), "Asymptotic expansions at work", Scandinavian Actuarial Journal, $1,143-152$.

Kent, J. T., Mardia, K. V., Rao, J. S. (1979), "A characterization of uniform distribution on the circle", Annals of Statistics, 7, 197-209.

Klugman, S. A., Panjer, H. H., Willmot, G. E. (2008), Loss Models: From Data to Decisions, 3rd Edition, Wiley \& Sons.

Kolesnik, A. D., Orsingher, E. (2005), "A planar random motion with an infinite number of directions controlled by the damped wave equation", Journal of Applied Probability, 42, 1168-1182.

Mardia, K. V., Jupp, P. E. (2000), Directional Statistics, Wiley.

Masoliver, M., Porrá, J. M., Weiss, G. H. (1993), "Some two and three-dimensional persistent random walk", Physica A: Statistical Mechanics and its Applications, 469-482.

Orsingher, E., De Gregorio, A. (2007), "Random flights in higher spaces", Journal of Theoretical Probability, 20, 769-806.

Srinivisan, R., Parthasarathy, S. (1976), Some Statistical Applications in X-ray Crystallography, Pergamon Press.

Stadje, W. (1987), "The exact probability distribution of a two-dimensional random walk", Journal of Statistical Physics, 46, 207-216.

Stadje, W. (1989), "Exact probability distribution for non-correlated random walk models", Journal of Statistical Physics, 56, 415-435.

Weiss, G. H., Kiefer, J. E. (1983), "The Pearson random walk with unequal step sizes", Journal of Physics, A: Mathematical and General, 16, 489-495. 